\title{
Roadmap on ultrafast optics
}

This content has been downloaded from IOPscience. Please scroll down to see the full text.

2016 J. Opt. 18093006

(http://iopscience.iop.org/2040-8986/18/9/093006)

View the table of contents for this issue, or go to the journal homepage for more

Download details:

IP Address: 130.183.90.175

This content was downloaded on 16/01/2017 at 15:10

Please note that terms and conditions apply.

You may also be interested in:

Single-pass high harmonic generation at high repetition rate and photon flux Steffen Hädrich, Jan Rothhardt, Manuel Krebs et al.

Introduction to macroscopic power scaling principles for high-order harmonic generation C M Heyl, C L Arnold, A Couairon et al.

Advances in attosecond science

Francesca Calegari, Giuseppe Sansone, Salvatore Stagira et al.

Design criteria for ultrafast optical parametric amplifiers

C Manzoni and G Cerullo

Continuous-wave femtosecond lasers

P G Kryukov

The birth of attosecond physics and its coming of age

Ferenc Krausz

The generation of ultrashort laser pulses

P M W French

Recent developments in fiber-based optical frequency comb and its applications

Wei Xia and Xuzong Chen

Fiber laser based hyperspectral sources

M E Fermann and I Hartl 


\title{
Roadmap
}

\section{Roadmap on ultrafast optics}

\author{
Derryck T Reid ${ }^{1,15}$, Christoph M Heyl ${ }^{2}$, Robert R Thomson ${ }^{1}$, Rick Trebino ${ }^{3}$, \\ Günter Steinmeyer ${ }^{4}$, Helen H Fielding ${ }^{5}$, Ronald Holzwarth ${ }^{6,7}$, \\ Zhigang Zhang ${ }^{8}$, Pascal Del'Haye ${ }^{9}$, Thomas Südmeyer ${ }^{10}$, Gérard Mourou ${ }^{11}$, \\ Toshiki Tajima $^{12}$, Daniele Faccio ${ }^{1}$, Frans J M Harren ${ }^{13}$ and Giulio Cerullo ${ }^{14}$
}

${ }^{1}$ Scottish Universities Physics Alliance (SUPA), Institute of Photonics and Quantum Sciences, School of Engineering and Physical Sciences, Heriot-Watt University, Riccarton, Edinburgh EH14 4AS, UK

${ }^{2}$ Department of Physics, Lund University, PO Box 118, SE-22100 Lund, Sweden

${ }^{3}$ Georgia Institute of Technology, School of Physics, Atlanta, GA 30332, USA

${ }^{4}$ Max Born Institute, Max-Born-Str.2a, 12489 Berlin, Germany

${ }^{5}$ Department of Chemistry, University College London, 20 Gordon Street, London WC1H 0AJ, UK

${ }^{6}$ Menlo Systems GmbH, Am Klopferspitz 19a, D-82152 Martinsried, Germany

${ }^{7}$ Max-Planck-Institut für Quantenoptik, Hans-Kopfermann-Str. 1, D-85741 Garching, Germany

${ }^{8}$ State Key Laboratory of Advanced Optical Communication System and Networks, School of Electronics Engineering and Computer Science, Peking University, Beijing 100871, People's Republic of China

${ }^{9}$ National Physical Laboratory (NPL), Teddington, TW11 OLW, UK

${ }^{10}$ Laboratoire Temps-Fréquence, Université de Neuchâtel, Avenue de Bellevaux 51, CH-2000 Neuchâtel, Switzerland

${ }^{11}$ IZEST, Ecole Polytechnique, F-91128 Palaiseau Cedex, France

${ }^{12}$ Department of Physics and Astronomy, University of California at Irvine, Irvine, CA 92697, USA

${ }^{13}$ Life Science Trace Gas Facility, Radboud University Nijmegen, PO Box 9010, NL-6500 GL, The Netherlands

${ }^{14}$ IFN-CNR, Dipartimento di Fisica, Politecnico di Milano, P.za L. da Vinci 32, I-20133 Milano, Italy

E-mail: D.T.Reid@hw.ac.uk

Received 13 January 2016, revised 5 May 2016

Accepted for publication 5 May 2016

Published 9 August 2016

\section{Abstract}

The year 2015 marked the 25th anniversary of modern ultrafast optics, since the demonstration of the first Kerr lens modelocked Ti:sapphire laser in 1990 (Spence et al 1990 Conf. on Lasers and Electro-Optics, CLEO, pp 619-20) heralded an explosion of scientific and engineering innovation. The impact of this disruptive technology extended well beyond the previous discipline boundaries of lasers, reaching into biology labs, manufacturing facilities, and even consumer healthcare and electronics. In recognition of such a milestone, this roadmap on Ultrafast Optics draws together articles from some of the key opinion leaders in the field to provide a freeze-frame of the state-of-the-art, while also attempting to forecast the technical and scientific paradigms which will define the field over the next 25 years. While no roadmap can be fully comprehensive, the thirteen articles here reflect the most exciting technical opportunities presented at the current time in Ultrafast Optics. Several articles examine the future landscape for ultrafast light sources, from practical solid-state/fiber lasers and Raman microresonators to exotic attosecond extreme ultraviolet and possibly even zeptosecond x-ray pulses. Others address the control and measurement challenges, requiring radical approaches to harness nonlinear effects such as filamentation and parametric generation, coupled with the question of how to most accurately characterise the field of ultrafast pulses simultaneously in space and time. Applications of ultrafast sources in materials processing, spectroscopy and time-resolved

15 Guest editor of the roadmap. 
chemistry are also discussed, highlighting the improvements in performance possible by using lasers of higher peak power and repetition rate, or by exploiting the phase stability of emerging new frequency comb sources.

Keywords: ultrafast optics, light sources, ultrafast control, time-resolved

(Some figures may appear in colour only in the online journal)

\section{Contents}

1. High-harmonic and attosecond pulse generation and spectroscopy 3

2. Ultrafast laser materials processing (LMP) 5

3. Frontiers and issues in the measurement of ultrashort laser pulses 7

4. Optical pulse characterization at the single-cycle limit 9

5. Time-resolved chemistry 11

6. Optical frequency combs (OFCs): an enabling technology 13

7. Ultrafast fiber laser sources 15

8. Microresonator sources of ultrafast pulses 17

9. Ultrafast solid-state laser sources 19

10. Compression of high energy pulses to the sub-attosecond regime: route to exawatt laser subatomic physics 21

11. Nonlinear propagation phenomena in ultrafast optics 23

12. Sensing and spectroscopy 25

13. Parametric frequency conversion 27 


\section{High-harmonic and attosecond pulse generation and spectroscopy}

\section{Christoph M Heyl}

Lund University

\section{Status}

During the last two (almost three) decades, ultra-short pulse extreme ultraviolet (XUV) sources based on high-order harmonic generation (HHG), have undergone continuous development. By focusing an intense laser pulse into a gas medium, researchers observed for the first time in 1987 weak high harmonic radiation. The underlying physical mechanism could be described by a simple model: an electron leaves the atom via tunnel ionization, is accelerated in the strong laser field and can then be driven back to the ion, where an XUV photon is emitted upon recombination. It was soon predicted, that this process could enable the generation of attosecond pulses, being emitted every half-cycle of the driving laser field. In 2001, the formation of attosecond pulse trains (the temporal analog of a high harmonic spectrum containing odd harmonics of the driving laser central frequency) could be demonstrated, followed by the first generation of isolated attosecond pulses (IAPs) [2].

Today's HHG-based XUV sources can provide $\mu \mathrm{J}$-level XUV pulses, they reach multi-MHz repetition rates, pulse durations below 100 as and photon energies spanning into the soft x-ray spectral region. These remarkable properties have enabled a wealth of scientific results and new experimental possibilities are continuously emerging. Today, a wide field involving ultrafast electron processes in various systems, ranging from ionization dynamics in simple atomic systems to charge migration tracking in complex molecules, is driving the research efforts of many groups in the attosecond and highharmonic research community. Moreover, coherent XUV sources employing HHG present a new class of table-top laserlike light sources. These sources allow extending various applications such as photoelectron spectroscopy on surfaces and interfaces, frequency comb (FC) spectroscopy (see also sections 8 and 12) as well as different nonlinear optical methods into a new photon energy regime, the XUV, which was previously not accessible with coherent table-top sources [3].

\section{Current and future challenges}

Many key techniques enabling attosecond spectroscopy and other applications involving HHG-based XUV sources have been demonstrated and new theoretical methods have been developed. It is todays challenge to further advance and apply these techniques, both within fundamental science as well as to problems of direct technological and interdisciplinary relevance. This includes further source development (see below), advances for the theoretical description of processes occurring at unfamiliar high photon energies and intensities as well as advances for many applications for which mainly conceptual development studies exist today. An interesting example is the exploration of ultrafast electronic and photonic signal processing possibilities and their ultimate limits [4]. While recent studies employing attosecond technology indicate such possibilities $[4,5]$, better control mechanisms and routinely applicable methods are needed in order make such schemes suitable for applications within modern electronics. Other few-fs and attosecond measurement approaches try to access ultrafast charge migration dynamics in molecules. First results indicate that such processes precede and possibly mediate structural changes in chemical reactions, opening a promising field, of importance within and beyond fundamental sciences [6]. For these two examples, the temporal properties of ultra-short XUV pulses are essential; other applications benefit mainly from other important pulse characteristics (see figure 1). Coherent imaging schemes for example, utilize the spatial coherence of the XUV light, enabling optical microscopy in the XUV regime, where the shorter wavelength makes it possible to reach high spatial resolution (also discussed in section 5). Especially with the prospect of reaching the water-window $(\sim 2.3-4.4 \mathrm{~nm})$, such approaches offer new possibilities for biological microscopy applications. However, the available photon flux of today's HHG-based XUV sources sets severe limitations [7].

\section{Advances in science and technology to meet challenges}

Progress in attosecond spectroscopy or, more generally, for many applications employing HHG based XUV sources, has and still is intimately connected to advances in attosecond source development. Despite their remarkable properties, HHG-based XUV sources suffer from one main issue: the low photon flux available in the XUV which is mainly limited by two factors, the average power of fs-laser systems and the conversion efficiency into the XUV. Both parameters are thus driving extensive development efforts. On the laser side, progress in optical parametric chirped pulse amplification technology is currently rapidly extending the available parameter regimes for laser-driven XUV sources (see also section 13). For HHG, strong efforts have been devoted to optimize the conversion into the XUV and to scale up the generated photon flux. However, the best values for gas-based HHG lie in the range of only $10^{-5}$ (considering the conversion from a driving laser pulse centered at $800 \mathrm{~nm}$ into one harmonic order around $20 \mathrm{eV}$ ), typically decreasing with increasing driving wavelength and increasing photon energy. For IAPs, the conversion efficiency is even smaller. A promising but challenging scheme for high-flux HHG (at high repetition rates) is the generation of high harmonic radiation inside an optical cavity [8] and very recent attempts point towards the application of this scheme for IAP generation [9]. As an alternative to gas-based HHG sources, other schemes employing surface and solid state-based HHG [5], light field synthesis [10] and free-electron lasing are emerging as complementary technology, especially for high-flux ultra-short XUV pulses.

Besides the efforts to increase the XUV photon flux, other important source development directions can be identified. Many applications set high demands on mainly one of the two parameters, pulse energy or repetition rate. 


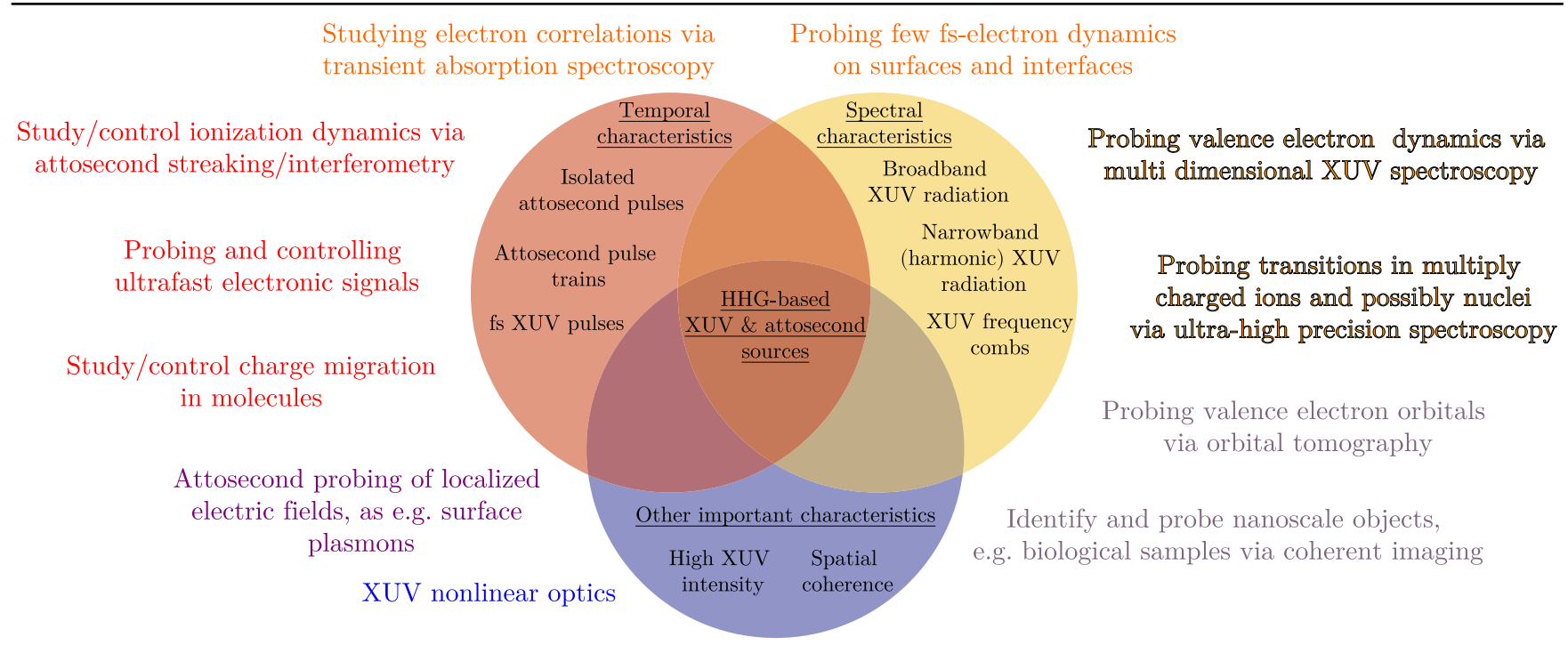

Figure 1. Current and future application directions for high-harmonic and attosecond sources, linked to the main source properties enabling the application.

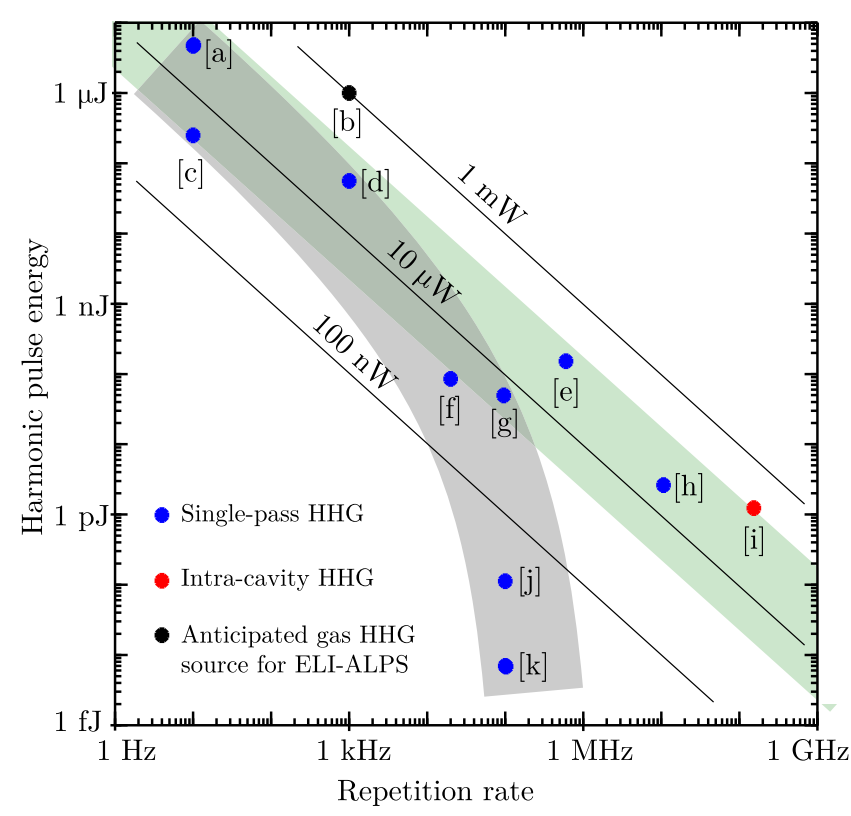

Figure 2. Generated pulse energy (in a single harmonic order above $20 \mathrm{eV}$ ), repetition rate and average power of state-of-the-art gas based HHG sources. References and details on the reported values: [a: see [14]], [b] expected parameter (a conversion eff. of $10^{-5}$ was taken into account), [c: see [15]], [d: see [16]], [e: see [17]], [f: see [18]], [g: see [19]], [h: see [20]], [i: see [8]], [j: see [21]], [k: see [22]]. The gray shaded area indicates a decreasing average power with increasing repetition rate, as reported in early experiments while recent results point towards constant average power/conversion efficiency (green shaded area).

Thus, even without pushing conversion efficiency or laser power further, high-harmonic and attosecond sources can still be scaled to either higher pulse energies, or higher repetition rates (see also figure 2), provided that suitable driving laser sources are developed. Recent results show that the required attosecond source scaling can be performed without changing the XUV generation characteristics [11], thus opening the possibility to further push attosecond sources in these directions.

Another important parameter for XUV sources is the photon energy. Today's IAPs sources typically span the spectral region around $20-100 \mathrm{eV}$. Extending IAP-sources (and more generally, sources approaching the attosecond regime) into the ultraviolet spectral range will provide new possibilities for ultrafast spectroscopy in solid state samples. Conversely, an extension to higher photon energies (see also section 10) [12], which goes along with the development of high-power long-wavelength laser sources, may make it possible to access even faster timescales [13].

\section{Concluding remarks}

With today's attosecond and HHG-based XUV source technology and the insight especially into fundamentally important electron processes these sources have enabled already, a wide and rapidly progressing field has been opened. This field offers many intriguing prospects such as the monitoring and control of reaction kinetics on an electronic level, ultrafast electronic and photonic signal processing, new perspectives for bio-microscopy as well as the direct access to transitions in highly charged ions and possibly even in nuclei (via HHG based XUV combs). The next decade will show whether attosecond and more generally, ultra-short pulse XUV science can meet these high expectations.

\section{Acknowledgments}

The author would like to gratefully acknowledge Anne L'Huillier for advise as well as support from the Swedish Research Council and the Knut and Alice Wallenberg Foundation. 


\section{Ultrafast laser materials processing (LMP)}

\section{Robert $R$ Thomson}

Heriot-Watt University

\section{Status}

Modern ultrafast lasers readily supply ultrashort laser pulses (femto- and picosecond) with peak-powers in the MW regime and higher. Such high peak-powers, on such short timescales, enable us to access highly nonlinear and non-equilibrium lightmatter interaction regimes during LMP. The high-peak powers facilitate nonlinear absorption of sub-bandgap photons. This enables LMP of high-bandgap materials (e.g. fused silica) using sub-bandgap light, and also the three-dimensional in-volume LMP, for applications such as optical waveguide writing, laserinduced chemical selective etching and material joining [23]. Compared to longer pulses, ultrashort pulse durations also enable a fundamentally different light - matter interaction during LMP, where energy is first deposited in the electronic subsystem, while the lattice remains effectively unaffected. This energy is then transferred to the lattice on longer timescales, after the pulse has left the focal volume. This decoupling of the energy deposition and lattice heating allows more efficient and spatially localized deposition of energy, minimizing the energy required to modify or ablate the material, minimizing collateral damage and the heat-affected zone.

Ultrafast LMP is becoming increasingly established in industrial laser-based production, for applications such as precision cutting of chemically toughened display glass [24], precision machining of holes in fuel injection nozels, and fragile parts such as biomedical stents [25, 26] (see figure 3-top). Femtosecond lasers are also becoming more common in the clinic, for the specific application of precision flap creation during laser-assisted in situ keratomileusis (LASIK). As shown in figure 3, this process utilizes the nonlinear absorption of focused femtosecond laser pulses to create a flap of epithelial material on the surface of the cornea. This flap can then be folded back to reveal the corneal stroma for shaping by a UV laser.

New applications for ultrafast LMP are now emerging. There are several companies offering products and services based on the capabilities of three-dimensional in-volume ultrafast laser inscription (ULI) (e.g. Translume, FemtoPrint, Optoscribe (of which the author is a co-founder), LightFab, Modular Photonics), where ultrashort pulses (usually $<1 \mathrm{ps}$ ) of sub-bandgap radiation are used to directly inscribe structural modifications inside a dielectric substrate. These modifications manifest themselves through changes to the refractive index and/or chemical etch rate. The chemical etch-rate modification capability can be used to fabricate structures such as microfluidics and micro-optics, and the refractive index modification can be used to fabricate devices such as three-dimensional optical waveguides. The waveguide writing capability is now finding applications in many areas, such as next generation spatially-multiplexed-telecommunications, where it is enabling the fabrication of devices such as integrated waveguide fan-outs [28] and photonic lanterns [29] for coupling light to and from multicore- and few-mode-fibers. A process

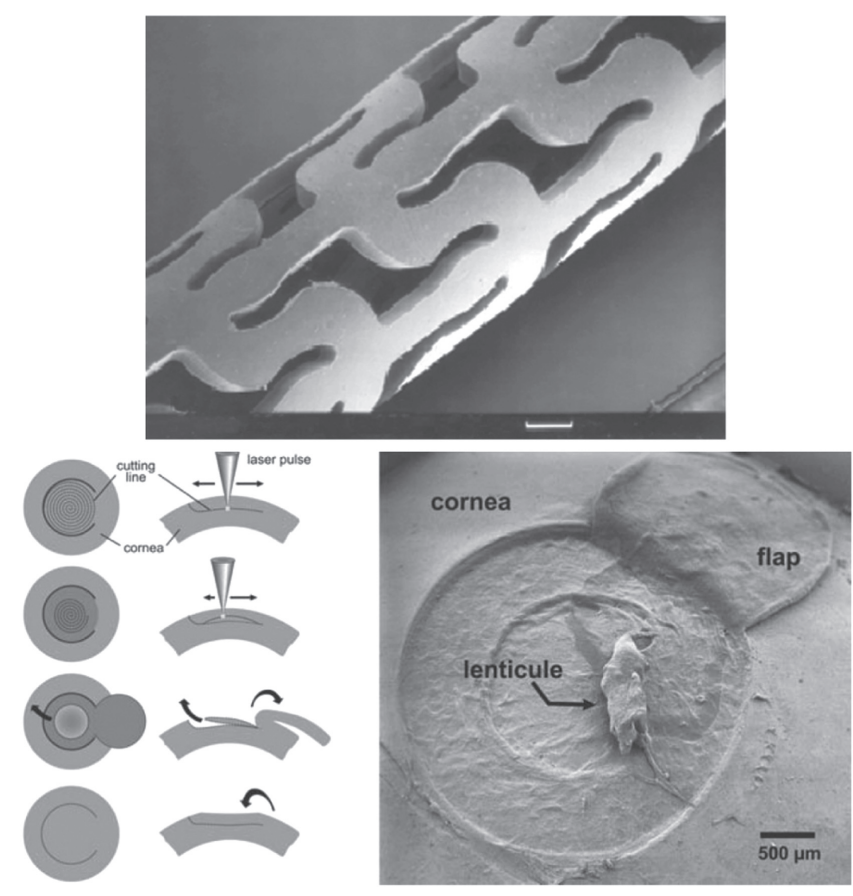

Figure 3. Top: biodegradable medical stent fabricated using ultrafast laser micromachining [26] (reproduced with permission from SPIE, copyright 2000). Bottom (left): steps involved in femtosecond laser based flap creation for LASIK. (Right): an scanning electron microscope image of a corneal flap [27]. Copyright 2000, with permission of Springer.

similar to ULI can also be used to 'weld' materials together, without a requirement for adhesives.

\section{Current and future challenges}

The full potential of ultrafast LMP can only be achieved if research continues across the full range of technology readiness levels. Basic research is essential to better understand the fundamental light-matter interaction processes during ultrafast LMP. Increased understanding would open up new capabilities, and enable the ultrafast LMP for a particular application to be intelligently optimized. More reliable and higher average power ultrafast laser sources are also required, as increasing the Watts $/ \$$ available from ultrafast lasers would also open up new mass-production applications. New beam manipulation technologies, such as scanners and spatial light modulators are also required, in order to efficiently use the increased average powers available from these sources. Fiber-optic beam delivery technologies are required, to deliver high-beam-quality and high peak power pulses. These would enable ultrafast welding processes to be performed without moving the part, a potentially important step when welding parts that require precision alignment. They would also enable the delivery of high peak power pulses into confined areas of the body, opening up new applications in medicine for precision tissue ablation.

Ultrafast lasers clearly present a unique and growing set of LMP capabilities, but it goes without saying that there is a considerable difference between demonstrating a technology in an academic environment, and proving its commercial and industrial viability. For ultrafast LMP to achieve its full 
potential, research must also target industrially relevant applications, and engage potential end users.

\section{Advances in science and technology to meet challenges}

One issue that has so far limited the industrial applications of ultrafast LMP is processing speed. New high average-power ultrafast laser systems are now coming to market and opening the way to increased throughput during ultrafast LMP. Trumpf now offer the TruMicro 5070 system, which delivers $100 \mathrm{~W}$ of $<10$ ps pulses at repetition rates of up to $600 \mathrm{kHz}$. For applications that require shorter pulses, there are also new higher average power fs-laser sources. Amplitude-Systemes (www.amplitudesystemes.com) supply the Tangor system which supplies a $300 \mathrm{kHz}$ train of $<500 \mathrm{fs}$ pulses with an average power of $>30 \mathrm{~W}$. Impressively, rhe InnoSlab system supplied by Amphos (www.amphos-usa.com) is capable of supplying $\mathrm{MHz}$ and $\mathrm{kHz}$ trains of sub-ps pulses at average powers in excess of $400 \mathrm{~W}$ !

The high average powers offered by these new systems can only deliver significantly higher LMP throughput if the power is properly utilized. One route to enable this is to use beam splitters or diffractive optical elements to split the beam into multiple beamlets (figure 4). Another approach is to utilize high-speed beam scanning technologies. For high numerical aperture applications, such as in-volume processing, high-speed galvo-scanning technologies have been developed, such as the high-speed microscanner from LightFab (www.lightfab.de). Depending on the device type and complexity, it has been demonstrated that combining a high average power ultrafast laser with a high speed galvo-scanner can reduce fabrication times by orders of magnitude, from hours to minutes. For low numerical aperture applications, such as surface machining and marking, high-speed polygonal scanners have been developed, such as the LSE300 from Nextscantechnology (nextscantechnology.com). This scanner can scan a large spot over a $300 \mathrm{~mm}$ field-of-view with a spot-scanning velocity of up to $100 \mathrm{~m} \mathrm{~s}^{-1}$. Another potential route to decrease fabrication times is to use a spatial light modulator to create multiple reconfigurable spots and hence more efficiently use of the power available from the laser [30, 31], but this will require high power handling technologies.

Medicine is one of the most exciting application areas for ultrafast LMP, with groups around the world investigating the potential of using ultrafast laser ablation in various areas of the body (e.g. eye and ear). The delivery of suitable laser radiation into these hard-to-reach regions is a significant technical challenge, but recent advances in novel hollow-core fibers, such as Kagome, Photonic Crystal, and Negative Curvature Fibers (NCF's) [32] now open the way towards a truly flexible and compact ultrafast laser scalpel suitable for endoscopic use. Fiberoptic delivery technologies such as these open the way to a plethora of exciting new medical applications for ultrafast LMP, as well as other applications in robotically controlled ultrafast LMP.
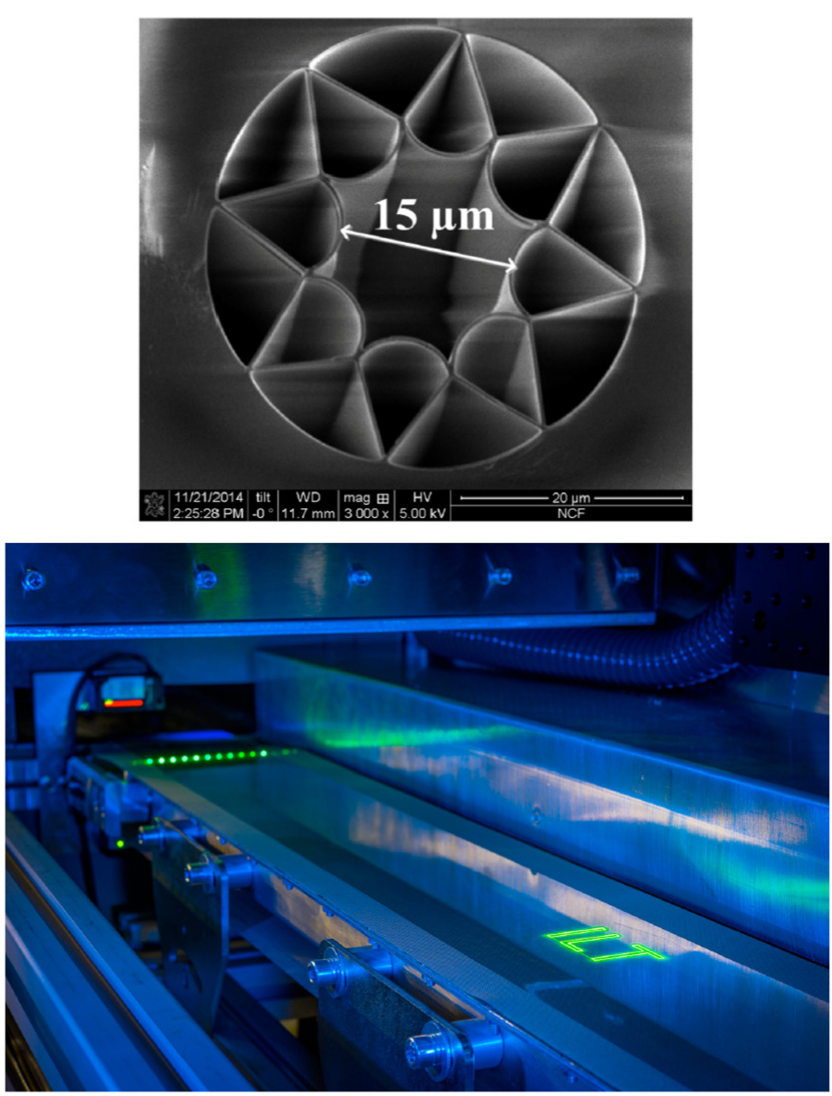

Figure 4. (Top) NCF for delivering ultrashort pulses [32]. (Bottom) Parallel ultrafast LMP of organic solar cells. (Reproduced courtesy of Dr-Ing. Arnold Gillner.)

\section{Concluding remarks}

The future for ultrafast LMP is bright, but challenges must be addressed to realize its full potential. New technologies, such as high average power lasers and high-speed scanners, will open up many industrial applications in mass-manufacturing, and high damage threshold hollow core fibers open up exciting applications in medicine. It is clear that ultrafast LMP is at an exciting transition, and it is now finding its niche in industry, yet it remains an active and high-impact area of both fundamental and applied academic research. With continued research, and targeted industry engagement, the applications for ultrafast LMP will continue to grow, with an anticipated benefit to science and society.

\section{Acknowledgments}

RRT acknowledges the STFC for support in the form of an Advanced Fellowship (ST/H005595/1), and via the STFCPRD (ST/K00235X/1) and STFC-CLASP (ST/K006509/1) schemes. RRT thanks the EPSRC for support through the EPSRC Centre for Innovative Manufacturing in Laser-based Production Processes (EP/K030884/1) and the IRC project Multiplexed 'Touch and Tell' Optical Molecular Sensing and Imaging (EP/K03197X/1). 


\section{Frontiers and issues in the measurement of ultrashort laser pulses}

\section{Rick Trebino}

Georgia Institute of Technology

\section{Status}

The field of ultrashort-laser-pulse measurement was born in the 1960s when laser pulses broke the nanosecond barrier and became shorter than could be measured using electronics. The first all-optical technique developed for such measurements, intensity autocorrelation, literally yielded only a blurry blackand-white image of the pulse in time and hence provided only a rough measure of the pulse length. Worse, for a complex pulse or unstable train of pulses, it yielded a very short spike atop a broad background. The spike, now generally referred to as the 'coherent artifact', indicated, not the pulse length, but the usually much shorter length of the shortest substructure in the pulse. Unfortunately, an autocorrelation's coherent artifact was-and still is-often confused for the actual pulse length, despite its correct interpretation in 1969 [35].

Today the field of pulse measurement is quite mature, and most common measurement problems have been solved. The frequency-resolved optical gating (FROG) class of techniques yield the complete temporal (and spectral) intensity and phase-literally a high-resolution color image of any pulse [36] - and accurately measure even very complex pulses. And due to its pulse-retrieval algorithm and over-determination of the pulse, FROG handles coherent-artifact problems much better than autocorrelation. When presented with an unstable train of pulses, FROG provides the correct approximate pulse length and a clear indication of the presence of instability [37]. Most FROG versions also provide a reasonable typical pulse when measuring an unstable pulse train. FROG operates over a wide range of pulse wavelengths, complexities, and lengths, from nanoseconds to attoseconds [38]. FROG also recently [33] achieved the single-shot measurement of extremely complex pulses (see figure 5) such as individual pulses of continuum and, potentially, optical rogue waves. And Akturk and coworkers showed that secondharmonic-generation FROG can measure pulses as short as one optical period in length, provided that a well-known geometrical-distortion-free crossed-beam geometry (in which the crystal is imaged onto a camera) is used [40].

Of course, FROG requires a spatially smooth beam and only measures a pulse in time and frequency, not space. But it provides a known reference pulse, required for potential spatiotemporal measurements.

It is important to mention that many alternatives to FROG have emerged and are also popular. However, many measure only the coherent artifact [37, 40] and so cannot distinguish between a stable train of short, simple pulses and an unstable train of long, complex pulses. While today's ultrafast lasers are often more stable than those of the 1960s, instability (for example, double-pulsing) can still occur. Also,
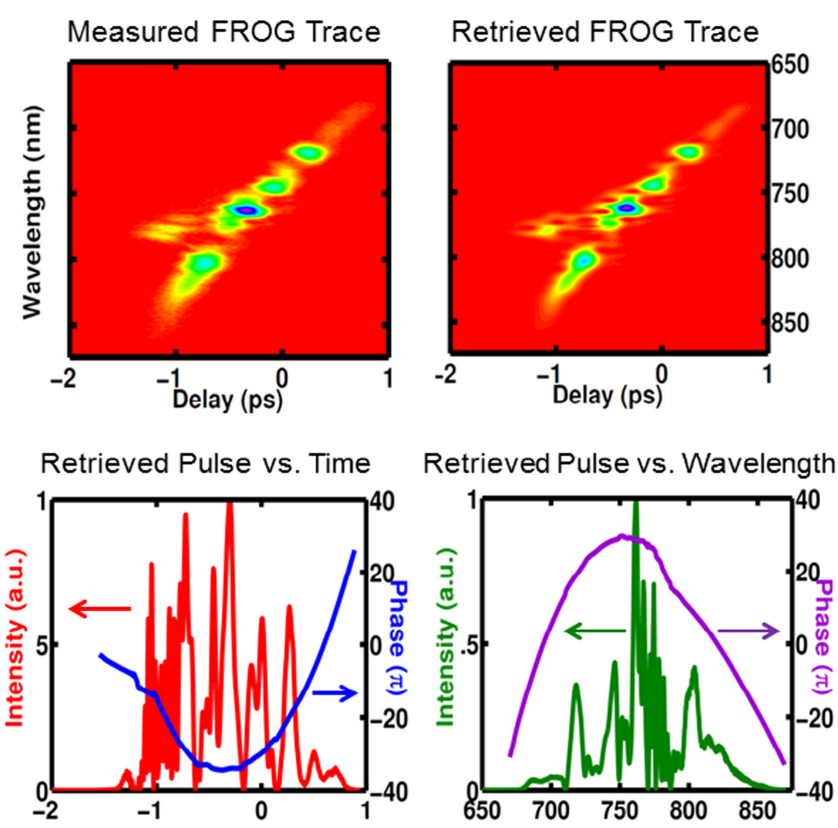

Figure 5. The first single-shot measurement of a complex continuum light pulse. It was achieved using the XFROG technique [33]. Reproduced with permission from [33] copyright 2014, OSA Publishing. Note the agreement between the measured and retrieved traces, a confirmation of the accuracy of the measurement (and, if this were a multi-shot measurement, the stability of the pulse train).

many utilize nonlinear-optical processes, which can also cause instability. As a result, users should exert care in using all methods (when every pulse in a train is different, no single result can be correct), but especially interferometric ones, to which instability is generally invisible. There is no independent 'ultrashort-pulse-train stability meter,' so this task necessarily falls to the pulse-measurement technique. Even if a method can, in principle, operate single-shot, in practice, it must usually operate multi-shot due to lasers' high repetition rates and low pulse energies. And true single-shot operation for a high-intensity pulse usually averages over a complex spatial mode - which is similar to multi-shot operation.

\section{Current and future challenges}

While many techniques have addressed the problem of measuring pulses in time and one spatial dimension, the main challenge currently facing the field of pulse measurement is the complete spatiotemporal measurement of pulses, yielding $E(x, y, t)$, especially on a single shot. As high-intensity laser systems generate ever increasing peak intensities and promise discoveries of new physics, undesired nonlinear-optically induced pulse distortions become more problematic. Such spatiotemporal distortions limit pulses' peak intensity and hence their utility, but currently go essentially unmeasured.

Even more inspiring is the possibility of illuminating a medium with an ultrashort light pulse and measuring the spatiotemporal field of the light back-scattered from it in order to determine the medium's properties. What medical diagnostics might arise from such a capability? Of course, this application will require a technique capable of measuring 
extremely complex speckle-like beams in space and time. Of course, the inverse numerical problem associated with such a measurement would also be extremely difficult.

Additional, less exotic, challenges include greater sensitivity, reduced device complexity, and, surprisingly, operation for longer (many ps) pulse lengths, which is actually challenging due to the relatively long delays and high spectral resolution required.

\section{Advances in science and technology to meet challenges}

Methods based on using a reference pulse (previously measured and established as stable) have begun to extend measurement techniques to, not just one, but two spatial dimensions as well as time. Using the SEA TADPOLE technique [34], which uses a near-field-scanning-opticalmicroscopy fiber to collect light to be measured, it is now possible to measure the complete spatiotemporal intensity and phase of pulses with femtosecond and submicron resolution (see figure 6). Another method, nanoFROG [41], has achieved similar resolution using a nanocrystal as the nonlinear-optical medium in a FROG. Both of these methods are, however, tedious to perform, requiring lengthy scans in space and producing four-dimensional data. Fortunately, phasediversity algorithms have significantly reduced the amount of data that must be taken [42], but such measurements still take too long (many minutes).

Single-shot complete spatiotemporal pulse measurement is the ultimate goal. At this time, only one such technique, called STRIPED FISH, has been proposed, and it is a promising approach. It uses a simple apparatus that generates multiple holograms at different wavelengths on a single camera frame [43]. It can measure a pulse with a space-timebandwidth product of as much as $\sim 1000000$ with currently available multi-megapixel cameras. While this seems to correspond to a very complex pulse in space and time, it is actually quite simple compared to pulses back-scattered from, say, a human body. Cameras with many more pixels would be very helpful in such measurements. Alternatively, speaking quite speculatively, multi-planar cameras whose different layers responded to different colors could revolutionize such measurements. On a different note, even the seemingly simple problem of displaying the results of such a measurement is also quite challenging. Software and/or display technology will have to be developed simply in order to display the pulse propagating in three-dimensional space and time.

Finally, extending these spatiotemporal techniques to attosecond pulses is important for this new field of science,

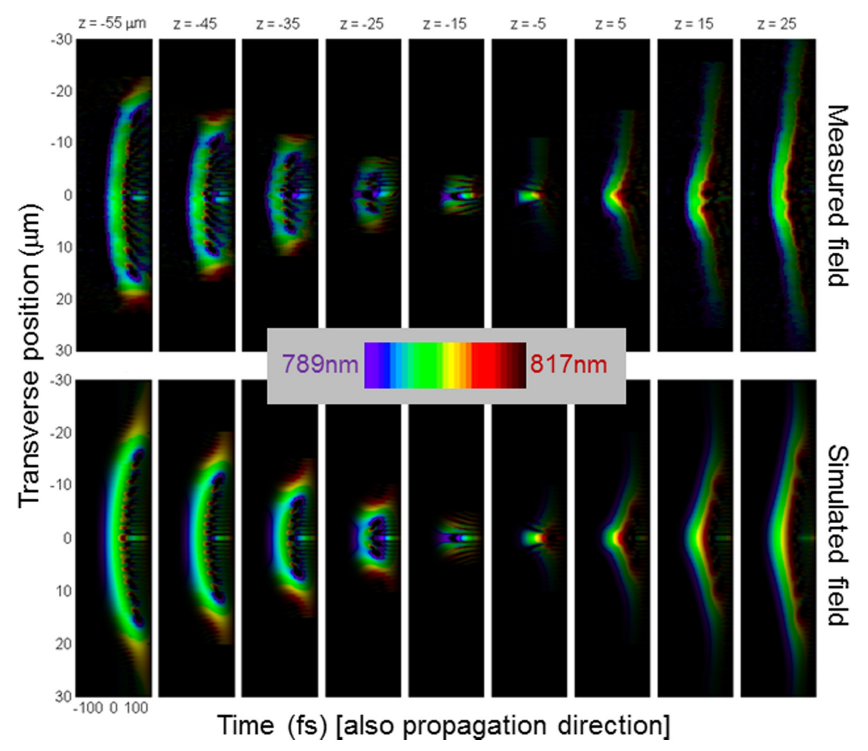

Figure 6. Spatiotemporal intensity and instantaneous frequency (plotted versus time and one transverse spatial coordinate), measured using the SEA TADPOLE method, of a pulse focused by an overfilled lens [34]. Reproduced with permission from [34] copyright 2014, OSA Publishing. Shown are nine snapshots of the pulse from the side as it propagates through the focus.

and XUV components will need to be developed in order to extend these simple visible/near-IR methods to this regime, even for simple pulses.

\section{Concluding remarks}

The field of ultrashort-laser-pulse measurement is a very mature field that has solved most of its most common problems. Of course, challenges remain, and they include the development of techniques for conveniently measuring the complete pulse spatiotemporal intensity and phase, $E(x, y, t)$, on a single shot and/or at exotic wavelengths, at attosecond pulse lengths, and for extremely complex pulses in space and time.

\section{Acknowledgments}

The author acknowledges support from National Science Foundation, Grant \#ECCS-1307817, and the Georgia Research Alliance. Rick Trebino owns a company that sells pulse-measurement devices. 


\section{Optical pulse characterization at the single-cycle limit}

Günter Steinmeyer

Max-Born-Institut, Berlin

\section{Status}

In the 1980s and earlier, the characterization of the shape and duration of an optical pulse exclusively relied on autocorrelation techniques. The dilemma of this approach lies in the fact that, as a matter of principle, one cannot reconstruct the actual underlying pulse shape from a measured autocorrelation.

Solution of this dilemma first came in the shape of FROG [36]. Spectrally resolving the autocorrelation (see figure 7) provides abundant information that enables unambiguous reconstruction of the pulse shape for almost all practical cases. Other techniques followed up, most notably interferometric approaches such as spectral phase interferometry for direct electric-field reconstruction (SPIDER [44]). SPIDER actually does not measure the temporal pulse shape, yet only the spectral phase, see figure 7. This measurement has to be combined with an independent measurement of the spectrum. Fourier transformation then provides the temporal pulse shape.

Other techniques further developed the ideas of the two prototypical complete pulse characterization techniques. FROG can be considered the paradigm of tomographic pulse characterization methods. While a FROG measurement requires scanning of the delay between two replicas of the pulse under test, one can equally well place a tunable filter at fixed delay in one arm of the correlator, which is known as the sonogram method, or one can completely abandon the correlator and replace it by a pair of adjustable wedges that allow to scan the group delay dispersion of the pulse, which is known as d-scan [45]. Another FROG-based development is known as multiphoton intrapulse interference phase scan [46], which involves a pulse shaper to actively manipulate and flatten the spectral phase of the pulse.

SPIDER has also inspired a number of variants. In its generic implementation, SPIDER only requires one-dimensional acquisition of interferograms whereas more advanced implementations of the SPIDER technique rely on spatially encoded arrangements of the SPIDER trace, which is referred to as SEA-SPIDER. A parallel development lead to a technique called 2DSI, which requires additional scanning of a delay while regular SPIDER requires no moving parts.

Today there is a zoo of methods, all fully capable of retrieving the amplitude and phase structure of ultrashort pulses. Nevertheless, there still is a remaining problem that is difficult to tackle. This is characterization of pulses that only consist of a single oscillation of the electric field.
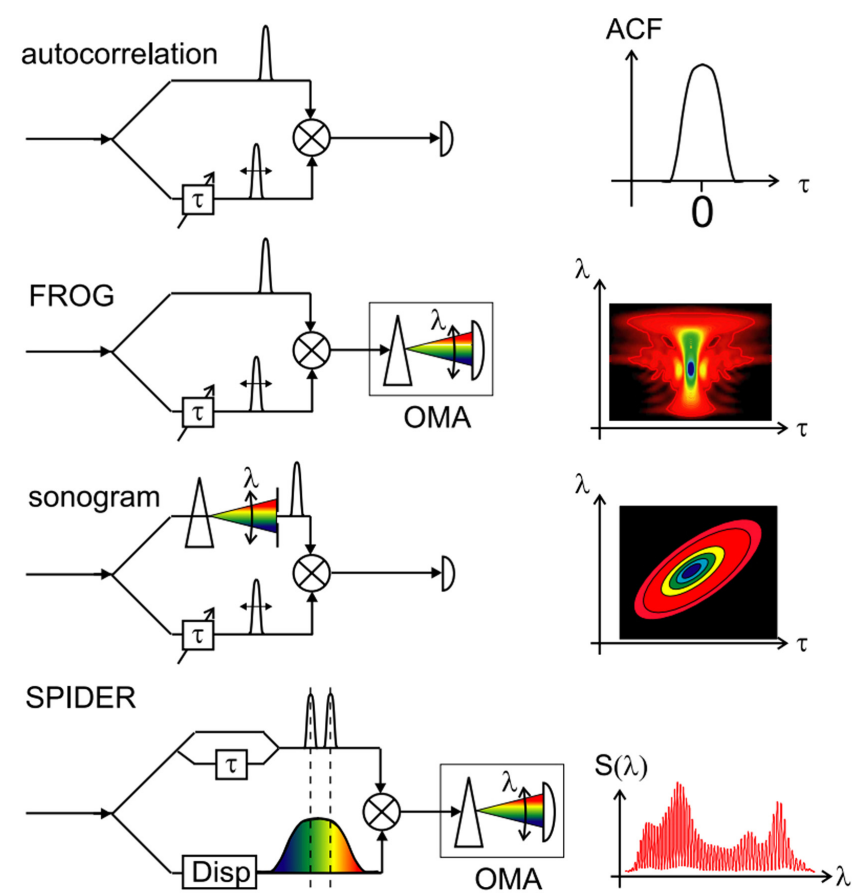

Figure 7. Schematic comparison of different measurement strategies, involving the generation of replica pulses, subsequent nonlinear optical processing, and spectral resolution. Reproduced with permission from [47].

\section{Current and future challenges}

When it comes to the characterization of extremely short optical pulses, all the above-mentioned techniques start to face extreme challenges. One ubiquitous problem is the bandwidth of the nonlinear optical process that is at the core of any pulse measurement techniques. Most implementations rely on second-order nonlinearities, i.e., second-harmonic or sum-frequency generation in nonlinear optical crystals like $\beta$ barium-borate (BBO). As wider bandwidths require shorter crystals, a natural limitation is typically reached at a crystal thickness of $10 \mu \mathrm{m}$, see figure 8 . For use of second-harmonic generation in FROG, this imposes a severe limitation for spectra that approach the octave. Sum-frequency generation with a monochromatic ancillary beam, as required in SPIDER, is more efficient in this regard, but also reaches limitations when more than octave coverage is required.

A second important aspect is the beam smearing problem [48]. Simply crossing two beams in a nonlinear medium makes the delay a function of lateral position in the crystal. Consequently, an autocorrelator measures a longer pulse in a non-collinear beam geometry than in a collinear one, i.e., a problem which is understood since the 1980s [49]. Fortunately, SPIDER is immune against beam smearing, and there exist implementations of FROG that are completely collinear, such as interferometric FROG. Finally, other variants of the tomographic methods like d-scan do not show this artifact either.

One can safely conclude at this stage that today complete pulse characterization variants exist which allow characterization of two-cycle pulses. However, let us now ask the 


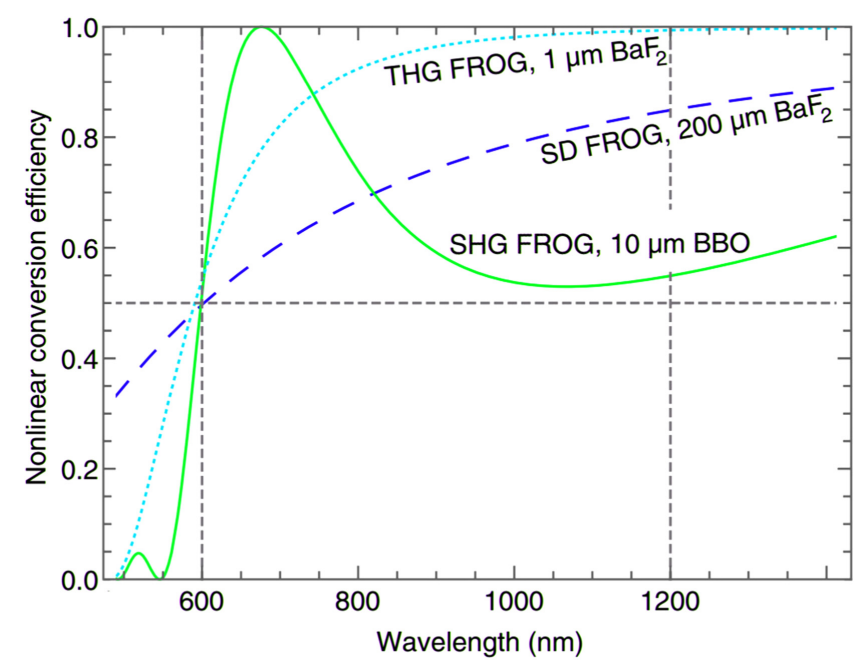

Figure 8. Obtainable phase matching bandwidth for the example of three different FROG variants (data from [50]). Self-diffraction enables use of a 20 times thicker nonlinear medium than secondharmonic generation.

question how far we can push techniques for reliable measurements of single-cycle or even sub-cycle pulses.

\section{Advances in science and technology to meet challenges}

The previous considerations clearly indicate conversion bandwidth of the nonlinear process as one particular bottleneck in accurately characterizing few-cycle pulses. One answer to this challenge could be the usage of surface nonlinearities like surface second-harmonic generation. A second possible method is the use of a four-wave mixing nonlinearity such as the self-diffraction process, see figure 8 . Here all input and the output wave share the same wavelength range, which reduces relative dephasing of the waves to an absolute minimum.

At the same time, however, both these possible solutions also impose restrictions on the beam geometry. Exploitation of surface nonlinearities requires tight focusing, which automatically disables non-collinear geometries as they are used in standard SPIDER or FROG approaches. Here interferometric FROG may be an answer. Then the advantage of identical wavelength ranges of the input and output beam immediately creates the problem on how to separate the output from the much stronger input. This can effectively only been done in a non-collinear geometry, and this immediately results in beam-smearing problems. While self-diffraction SPIDER poses a lot of additional challenges of its own [50], it may well be one of the few possible ways to go for the measurement of sub-cycle pulses.

Given the severe constraints of traditional characterization techniques, maybe one needs do something radically different at extremely short pulses durations? The answer to this question may be stereo above-threshold ionization (stereo ATI [51]). This method is directly sensitive to the oscillating electric field, rather than to the intensity envelope. When pulses approach the single cycle limit there may be a substantial asymmetry between the maximum negative and maximum positive electric field within a short pulse. This asymmetry can be used to estimate the duration of the short pulse. And while stereo ATI is far away from being a full characterization method, it may actually be the best bet for measuring the shortest pulses.

\section{Concluding remarks}

Pulse characterization has certainly come of age, with methods like FROG and SPIDER that enable access to the complete amplitude and phase information of femtosecond pulses. While pulse generation techniques are advancing into the single-cycle regime, this poses new challenges for pulse measurement techniques. However, there are a few promising approaches that may be able to overcome the single-cycle barrier. Currently, one of the best ways appears combination of several parallel measurement techniques [51] as, for example, SEA-SPIDER and stereo ATI. 


\section{Time-resolved chemistry}

\author{
Helen H Fielding
}

University College London

\section{Status}

Breaking and making molecular bonds is fundamental to chemistry. The typical vibration period of a covalent bond in a molecule is of the order of tens of femtoseconds; therefore, to observe bond rearrangements during chemical reactions requires us to probe them on this timescale. Ultrafast measurements of chemical reactions became possible in the late 1980 s, with the advent of femtosecond lasers. The first pioneering experiments using femtosecond lasers to record 'molecular movies' of bonds being broken and made were carried out by Ahmed Zewail and coworkers; the significance of this work was recognized by the award of the Chemistry Nobel Prize in 1999 [52].

Femtosecond laser experiments for time-resolved chemistry employ pump-probe techniques in which a pump laser pulse initiates a chemical reaction, or creates a non-stationary state or wave packet, and a probe laser pulse is fired at a series of precisely timed intervals after the pump pulse. The probe laser pulse is configured to generate an observable that provides information about the evolving system. Precisely what information is obtained from these experiments is dependent on the pump-probe excitation scheme and the observable. During the last two decades, a great deal of progress has been made in developing differential detection techniques that allow us to obtain detailed information about the pathway from reactants to products.

Femtosecond time-resolved photoelectron spectroscopy (TRPES) has proved to be a powerful detection technique for probing ultrafast molecular dynamics in the gas phase [53], for example, to probe the photoresponse of the green fluorescent protein chromophore (figure 9) [54]. The related techniques of femtosecond time-resolved photoion spectroscopy and photoelectron-photoion coincidence spectroscopy have proved invaluable for unraveling the mechanisms of photoinduced fragmentation reactions [55]. In solution, femtosecond dispersed fluorescence spectroscopy and femtosecond transient absorption spectroscopy have been shown to be particularly valuable tools and have allowed, for example, the observation of unidirectional rotation in lightdriven molecular motors [56] and simple bimolecular reactions [57].

The next step is to advance these tools to follow bond rearrangements directly, in larger molecules and bimolecular reactions, in complex chemical and biological systems and advanced materials, in the same level of detail that is currently possible for small isolated molecules.

\section{Current and future challenges}

In polyatomic molecules, ultrafast non-adiabatic transitions through conical intersections connecting the potential energy

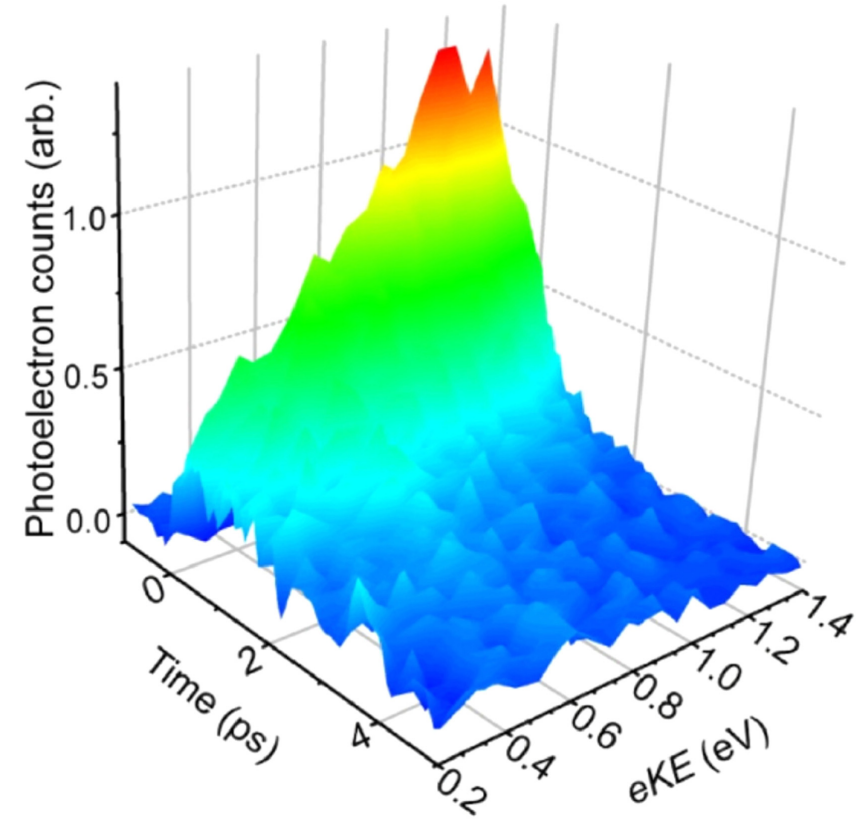

Figure 9. Experimental photoelectron spectra of the deprotonated green fluorescent protein chromophore as a function of pump-probe delay, following excitation of the $S_{1}$ state at $500 \mathrm{~nm}$ and probing at $800 \mathrm{~nm}$. Reproduced with permission from [54], copyright 2013 Royal Society of Chemistry.

surfaces associated with different electronic states are ubiquitous. One of the current challenges is to track wave packet motion through these conical intersections directly. Not only is the motion very fast (typically $10 \mathrm{~s}$ fs) but to ionize the lower lying electronic states of neutral molecules tends to require vacuum ultraviolet (VUV) light. UV-pump VUV-probe TRPES using sub-20 fs laser pulses is a promising tool for following ultrafast nonadiabatic dynamics in large molecules [53]. Photoelectron circular dichroism (PECD) using circularly polarized light sources has been shown to be a sensitive probe of chirality and femtosecond pump-probe PECD shows promise as a tool for probing the dynamics of large systems due to the sensitivity of PECD to molecular conformation [58].

In solution, current challenges include observing the solvent response to photoexcitation of a solute and distinguishing dephasing and ensemble averaging contributions to decoherence times. Multidimensional spectroscopies are powerful tools for identifying vibrationally, electronically or vibronically coupled states and will play an important role in unraveling the complex spectra of larger systems. The introduction of liquid microjets has made it possible to extend photoelectron spectroscopy of molecular systems to the solution phase [53]. So far, femtosecond TRPES studies in liquid jets have focused on solvated electrons and small biomolecules and the challenge is to extend this technique to complex systems, such as proteins.

Recent developments in the generation of ultrashort pulses of photons with very short wavelengths (see section 1) are opening up unprecedented new opportunities for chemical dynamics [59]. Femtosecond slicing methods at synchrotrons are enabling $\mathrm{x}$-ray absorption and emission spectroscopies 
and $\mathrm{x}$-ray free electron lasers (XFELs), which have photon fluxes that are several orders of magnitude higher, represent a real revolution in the field of structural dynamics. A major challenge is to extend successful picosecond pump-probe $\mathrm{x}$-ray studies at 3rd generation synchrotrons to the femtosecond time regime at XFELs and combine sub-Angstrom spatial resolution for mapping individual atoms with sub100 fs temporal resolution. X-ray crystallographic resolution of small photoinduced differences, in the photoactive yellow protein, has been shown to be possible [60], and the first molecular movie using $\mathrm{x}$-ray scattering, for the electrocyclic ring opening reaction of 1,3-cyclohexadiene to 1,3,5-hexatriene [61], has been reported (see figure 10). The next challenge is to study more complex chemical and biological reactions in the condensed phase.

Another challenge is the coherent control of electron motion and bond formation, which might eventually open up the possibility for industrial scale photochemical synthesis.

\section{Advances in science and technology to meet challenges}

Although synchrotrons and XFELs provide the potential for studying dynamics of chemical and biological systems with unprecedented time and space resolution, there are many technical challenges. These include improving methods of sample delivery for complex chemical and biological systems and advanced materials, improving the stability of the light sources, such as fluctuations in XFEL spectra and pulse-topulse intensity and pump-probe time-jitter, developing ultrafast x-ray optics, such as split-and-delay optics, high dynamic range diagnostics and high frame-rate and dynamic range area detectors. Increasingly sophisticated measurements also require more sophisticated numerical tools to deal with data processing at high spatial and temporal resolution.

Interpreting the results of more differential measurements on more complex systems requires sophisticated computational methods capable of solving the time-dependent Schrödinger equation at the atomic level at very small time intervals. A major challenge for computational excited state dynamics is to combine accurate potential energy surfaces with accurate nuclear dynamics. Current methods are computationally expensive and often not stable over a range of configurations and become even more complicated in an environment. Another major challenge is the need to calculate experimental observables directly.

The development of truly broadband visible and IR sources will open up the possibility for carrying out broad

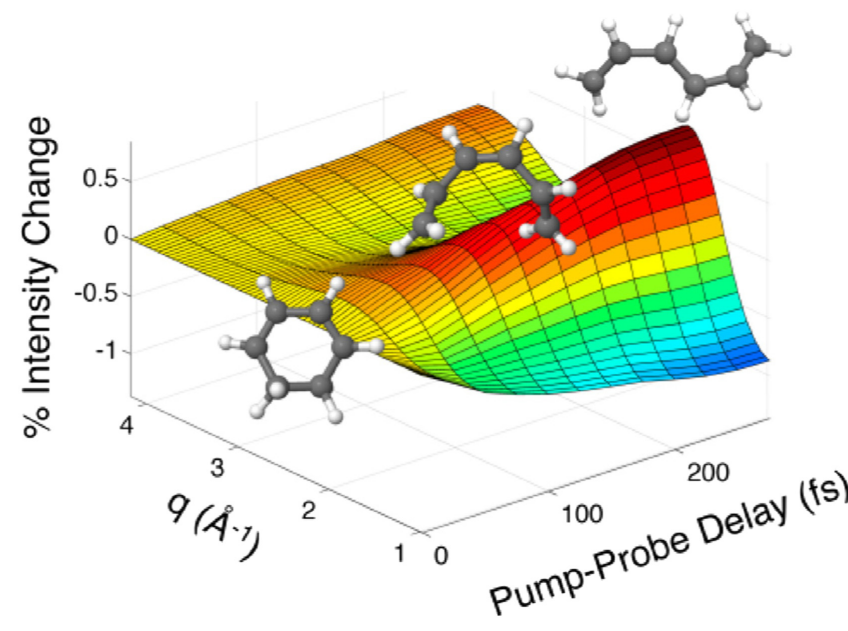

Figure 10. Calculated \% intensity change in x-ray scattering pattern during the ring opening reaction of 1,3-cyclohexadiene as a function of pump-probe delay [61]. Adapted with permission from [61], copyright 2015 American Physical Society.

survey multidimensional spectroscopies in solution to identify coupled modes rapidly. A challenge might be to develop standard pulse sequence designs and pulse shapers and standard software packages for analysis and interpretation (see section 3), so multidimensional spectroscopy could become a routine tool for chemists, like multidimensional NMR.

\section{Concluding remarks}

Understanding physical, chemical and biological change at the microscopic scale is important in a diverse range of processes in nature and technology, from light harvesting and photodynamic therapy to nanoscale machines and electronic devices. A major goal is to develop our understanding to the point where it becomes possible to design molecular systems and light fields, for a particular function, from first principles. The possibility of increasingly differential measurements and calculations on more complex molecular systems, makes the future for femtosecond chemistry very exciting.

\section{Acknowledgments}

I would like to thank the following colleagues for their valuable contributions: Jason Greenwood, Adam Kirrander, Stephen Meech, Russell Minns, Andrew Orr-Ewing, Michael Robb, Katharine Reid, Jonathan Underwood, Jasper van Thor and Graham Worth. 


\section{Optical frequency combs (OFCs): an enabling technology}

\section{Ronald Holzwarth}

Menlo Systems GmbH and Max-Planck-Institut für Quantenoptik

\section{Status}

OFCs generated by ultrafast mode-locked femtosecond (fs) lasers have since their first demonstration in 1998 [62] revolutionized the art of measuring and controlling the frequency of light waves. They provide us with a series of precisely spaced, sharp spectral lines and can be seen as a ruler in frequency space or as a gear for dividing down or multiplying up to optical frequencies of hundreds of $\mathrm{THz}$. Today they are used in various applications such as precision spectroscopy and optical atomic clocks, distance measurements, trace gas sensing and related environmental science applications, ultracold gases, molecular fingerprinting and associated biomedical applications, precision spectroscopy in astronomy and attosecond physics, to name a few.

For most applications today, the OFC is generated by a mode locked fs laser. To understand its mode structure, we consider a pulse circulating in a laser cavity with length $L$ at a carrier frequency $f_{\mathrm{c}}$ that is subject to amplitude modulation described by an envelope function $A(t)$ with periodicity $T$ given as the pulse round trip time $T=2 L / v_{\mathrm{gr}}$. Fourier transformation of $A(t)$ reveals that the resulting spectrum consists of a comb of laser modes separated by the pulse repetition frequency $f_{\mathrm{r}}=1 / T$ and centered at $f_{\mathrm{c}}$. Since $f_{\mathrm{c}}$ is not necessarily an integer multiple of $f_{\mathrm{r}}$, the modes are shifted from being exact harmonics of the pulse repetition frequency by an offset $f_{\mathrm{o}}$ (see figure 11):

$$
f_{\mathrm{n}}=n \times f_{\mathrm{r}}+f_{\mathrm{o}}, n=\text { a large integer. }
$$

This equation maps two radio frequencies $f_{\mathrm{r}}$ and $f_{\mathrm{o}}$ onto the optical frequencies $f_{\mathrm{n}}$. While $f_{\mathrm{r}}$ is readily measurable, $f_{\mathrm{o}}$ is not as easily accessible. In the most simple case that an octave spanning optical spectrum is available an $f: 2 f$ interferometer can be used to detect the offset frequency. Over the years fs laser sources based on different gain materials have been used for comb generation. Early work has mainly been done with Ti:Sa lasers, today much of the work is done with fiber lasers based on $\mathrm{Er}, \mathrm{Yb}$ or Tm doped fibers.

Since 2007 a new OFC generation scheme has emerged that uses parametric frequency conversion in micro resonators with very high quality factors $(Q)$ [63]. Such micro resonator optical frequency combs (MFC) — or Kerr combs-are generated by coupling a continuous wave pump laser into a high $Q$ optical micro resonator with a Kerr nonlinearity which converts an input pump laser to an OFC via parametric processes. MFCs generate the same equidistant grid of optical frequency components as fs laser based combs, provided that the parameters are chosen correctly. This approach allows access to high repetition rates in the range of $10 \mathrm{GHz}-1 \mathrm{THz}$

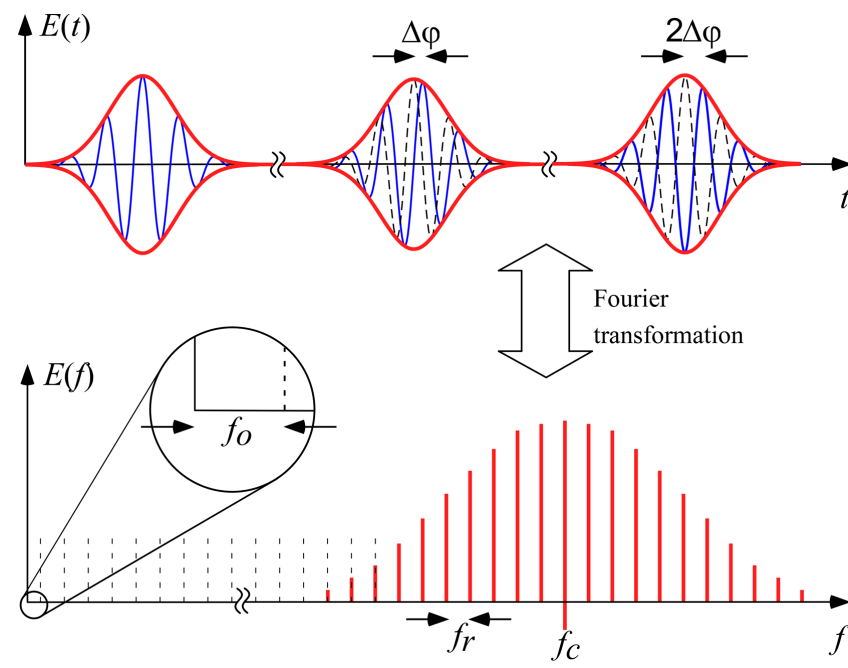

Figure 11. Optical pulse train in time and frequency domain. The offset frequency is linked to the pulse to pulse phase slip between carrier and envelope of the pulse by $f_{0}=\Delta \varphi / T 2 \pi$.

not easily accessible with mode locked lasers, facilitated by the small dimensions of typical resonator designs.

Already before the invention of fs laser based comb sources, combs based on electro optic modulation have been used [64]. Such devices have lately been brought to new heights and direct $f: 2 f$ stabilization has become possible. On the other hand, active modulation also allows mode locking of compact diode laser sources, including quantum cascade lasers (QCL).

\section{Current and future challenges}

OFCs have in the course of the past almost two decades found their way into a diverse number of applications. Each of these applications poses its own set of requirements and challenges.

The original driver to develop OFCs has been precision spectroscopy on atomic hydrogen. As of today, this is a very active field of research, serving as a test bed for quantum electrodynamics. Precision spectroscopy at the extreme has led to the current generation of optical clocks now reaching the low $10^{-18}$ level not only in terms of stability but also accuracy [65]. This development satisfies the ever increasing demand for stability and accuracy of time and frequency signals. Such optical clocks rely on narrowband optical transitions and use a OFC as clockwork. Therefore OFCs need to keep pace. A high shortterm stability of the OFC is required to shorten measurement time and to ease the process of investigating systematic uncertainties and drifts. To this end phase drifts within the combs system have to be analyzed and eliminated to the highest degree possible. RF signals from down-converted optical oscillators can serve as generators for low phase noise microwave signals that serve as sources for radar or astronomical VLBI applications.

In recent years, OFCs have also found their way into astronomy, as calibrators for astronomical spectrographs. Their unparalleled accuracy opens up new areas, such as the detection of Earth-like extrasolar planets through radialvelocity measurements or the direct observation of the 
accelerated cosmic expansion. The regularly spaced lines of an OFC that are well known and controlled by an atomic clock are ideally suited to calibrate spectrographs, surpassing common thorium-argon calibration lamps in many ways [66]. The challenge here is that the comb lines must be resolved by the spectrograph, which requires a large mode spacing of typically $15-30 \mathrm{GHz}$ and a broad spectral coverage is paramount, preferably the full visible and near infrared spectral range from 380 to $1200 \mathrm{~nm}$. In order to be useful tools for astronomers they have to be operated remotely and continuously, requiring engineering questions to be solved.

Biomedical optics and molecular spectroscopy drive the development towards mid-IR wavelengths. Commonly used Fourier transform spectroscopy (FTS) has shortcomings in terms of resolution and acquisition speed that can be overcome with OFC based techniques [67]. Other applications requiring coherent XUV light can also benefit from OFCs. For the OFCs to be useful in the frequency domain they have to be generated with multi MHz mode spacing. This results in a reduced energy per pulse, which is usually offset by adding an enhancement cavity around the noble gas jet used for high harmonic generation (HHG) [68].

\section{Advances in science and technology to meet challenges}

The advances in the last few years to meet these challenges fall basically into three categories.

Combs generated by micro resonators are finally well enough understood to allow reproducible low noise operation and self referencing, the well known fs laser OFCs have made big technological progress towards low noise, easy to use, space and airborne systems and finally a lot of technology has been developed around the combs to convert the combs into the UV and mid-IR spectral range and use dual comb systems for different applications.

Micro resonator based combs investigated around the world mainly fall into two platforms: crystalline or amorphous material (e.g. $\mathrm{MgFl}$ or fused silica) micro-machined and polished into circular geometry and on chip waveguide based devices (e.g. SiN). With the first showing higher $Q$, the latter showing lower $Q$ but higher nonlinearity. Compactness and possibly chip-scale integration together with the high repetition rates in the tens of $\mathrm{GHz}$ range is enabling an increased number of comb applications, especially in astronomy, microwave photonics, or optical telecommunications. Although the technology is somewhat less mature than the laser based combs, big advances have been made in the past two years in understanding the noise sources, allowing reproducible low noise operation, including the generation of solitons, as well as self referencing of MFC systems [69]. MFC generation is also possible in the mid-IR, currently a very active field of research.

Femtosecond laser-based combs are the work horses for most OFC applications around the world. Solid-state as well as fiber laser sources are investigated. Fiber lasers having the advantage of better usability and ruggedness, solid-state lasers with their higher cavity finesse are less prone to ASE and pump noise. Fiber lasers with increased control bandwidth and orthogonalized electro optic actuators are now rivaling solid-state based combs for best performance. Higher control bandwidth resulting in very tight locks to optical references and all polarization independent fiber laser designs using nonlinear optical loop mirrors (NOLM) make this possible. Stability and accuracy of such systems is below $1 \times 10^{-16}$ at $1 \mathrm{~s}$ and well below $10^{-18}$ at $1000 \mathrm{~s}$, the benchmark of current optical clock development [70]. Other applications as envisioned in mission scenarios for future ESA and NASA missions require OFCs to be space proof or ready for airborne use. First steps in this direction have been taken, with suborbital sounding rocket flights in April 2015 and January 2016, bringing a fully functional OFC into space and back [71].

Technology development around OFCs is currently experiencing an unprecedented boost. Firstly, this boost is targeted towards wavelength conversion to other, previously not accessible wavelength. High harmonic generation in gas jets inside enhancement resonators has finally produced remarkable results, generating XUV combs with power levels useful for spectroscopic applications. On the mid-IR side of the spectrum, FCs are now routinely generated either by difference frequency generation (DFG) that produces offset free combs or by optical parametric oscillators (OPOs). The later is especially useful for average powers at the Watt level. In the mid-IR spectral range, OFCs generated by frequency modulated QCLs [72] are currently receiving a lot of attention. Another branch that has lately attracted a lot of attention is the use of two FCs with slightly offset repetition frequencies. Such a configuration is the comb equivalent to classical FTS, with the advantage of fast acquisition times of a few $10 \mu \mathrm{s}$, broad spectral coverage and unprecedented accuracy.

This is certainly a non-exhaustive list of activities that has seen a steady growth over the last years.

\section{Concluding remarks}

OFCs today are a mature, commercially available technology. Nevertheless, we still see an ever increasing number of R\&D activities from various fields all around the world, making the OFC community a very active one and OFCs a truly enabling technology.

Many of these OFC applications are ready to leave the optical table inside well controlled laboratory environments enabling unprecedented measurement capabilities in the field.

\section{Acknowledgments}

I would like to thank the comb teams with all current and former members at MPQ and Menlo for many exciting and stimulating years of joint work. 


\section{Ultrafast fiber laser sources}

Zhigang Zhang

Peking University

\section{Status}

Benefitting from the wave guiding property of an optical fiber, fiber lasers have the intrinsic advantages of excellent heat dissipatability, near diffraction-limited beam quality, large gain, compactness and alignment robustness. Those fascinating features have motivated researchers to use fibers as ultrashort pulse generation devices for more than two decades [73].

There have been three mode-locking techniques developed for fiber lasers: the nonlinear amplifying loop mirror (NALM), the nonlinear polarization evolution (NPE) and the saturable absorbers. The NALM is the earliest mode-locking technique in Er:fiber lasers. However, the mode-locking is not self-starting. Shaking mirrors or saturable absorbers have to be applied to assist mode-locking. Since the NPE technique was developed, mode-locking can be self-starting. Semiconductor saturable absorber mirrors (SESAMs) are the most successful among all saturable absorbers. The SESAM can be used either solely in linear cavity fiber lasers or in NPE ring lasers as a mode-locking starter. Recently, novel materials as mode-locking devices, such as carbon nanotube and graphene saturable absorbers, have become popular research topics. At the same time, the new point of view on NALM has triggered the renaissance of the so called 'figure 8' fiber laser (F8L, the joint double-ring fiber cavity looks like the number ' 8 ') [74]. The enlarged splitting ratio in the coupler makes the fiber laser work at a higher initial intracavity power, so that there are more opportunities to generate high power spikes for the nonlinear loop mirror to catch. The polarization maintaining fiber keeps the mode locking robust once it builds up. This is particularly important in industrial applications such as material processing, introduced in section 2.

For shaping of the intracavity pulses, the key process is the interplay between the dispersion and the nonlinearity. Soliton pulse shaping is the primary process that dominated the early fiber lasers at communication wavelength. As the normal dispersion takes part in the process, the pulse profile greatly varies along the fiber depending on the amount of the normal dispersion. The dispersion managed fiber laser as it is called can manage the net fiber dispersion for from anomalous to all-normal so that the lasers are still mode locked. In the allnormal dispersion regime, the key to keep the laser working in pulsed mode is the spectral filtering that prevents the pulse from further spreading by chopping the leading and falling parts of the pulse.

Ultrafast fiber lasers are becoming the fundamental elements in many optical systems for numerous industrial, medical and scientific applications. Intense pulse applications such as coherent $\mathrm{x}$-ray and attosecond pulse generation and laser-driven particle accelerators, normally operated with solid-state lasers, also expect to use fiber lasers that can be generated by coherent combination of a bundle of fiber output.

\section{Current and future challenges}

Current challenges in mode-locked fiber lasers are still the reliable mode-locking starting and pulse shaping mechanisms, pulse energy, high order dispersion compensation in amplified pulses, wavelength extension, novel fibers for fiber lasers and pulse delivery and coherent beam combination.

\section{Reliability}

Although carbon nanotubes and graphene have demonstrated successful mode-locking devices, the uncertain modulation depth, sophisticated preparation process, large non-saturable loss and low damage threshold are still the key issues that block their real-world application in mode-locking fiber lasers. New materials towards a faster recovery time, a lower non-saturable absorption loss and a higher damage threshold are the challenges. On the other hand, searching for novel mode-locking mechanisms without using saturable absorber materials is also challenging.

\section{Pulse energy and peak power}

Pulse energy is one of the main parameters that fiber lasers are behind on bulk lasers described in section 7. To increase the pulse energy, the most straightforward way is to increase the mode field area of the fiber. To date, the largest fundament mode area is around $50000 \mu \mathrm{m}^{2}$ [75] for un-doped fibers, and for doped fibers it is in the range of $5000-8000 \mu \mathrm{m}^{2}$ [76]. However, even with unlimited mode area the ultimate limitation is the self-focusing effect inside the fiber which will limit the peak power of fiber laser operating in the fundamental mode to around $6 \mathrm{MW}$ [77].

\section{Repetition rate}

The pulse repetition rate of fiber lasers draws attention mainly due to the requirement in the OFC generation and industrial applications. $1 \mathrm{GHz}$ repetition rate fiber laser has been demonstrated [78] with innovative high power integrated WDM-collimtors (figure 12). The challenge is how short the fiber can be for the highest fundamental repetition rate to reach multi-gigahertz with silicate fiber. Highly doped fiber can only be seen in phosphate fiber [79]. On the other hand, a repetition rate below $1 \mathrm{MHz}$ required in micro-machining is also a challenge due to the difficulty in pulse shaping in super long fiber cavity.

\section{Wavelength range expansion}

Because of the growing interest of mid-infrared (MIR) laser sources for applications in attosecond and $\mathrm{x}$-ray science, molecular spectroscopy and coherent control, it is required to extend the fiber laser to run at longer wavelengths. The transmission of fused silica glass is below $2.3 \mu \mathrm{m}$ [80]. To further extend the operating wavelength, it is necessary to use a non-oxide glass, such as fluoride or sulfide glass. There have been many fiber lasers based on fluoride glass 


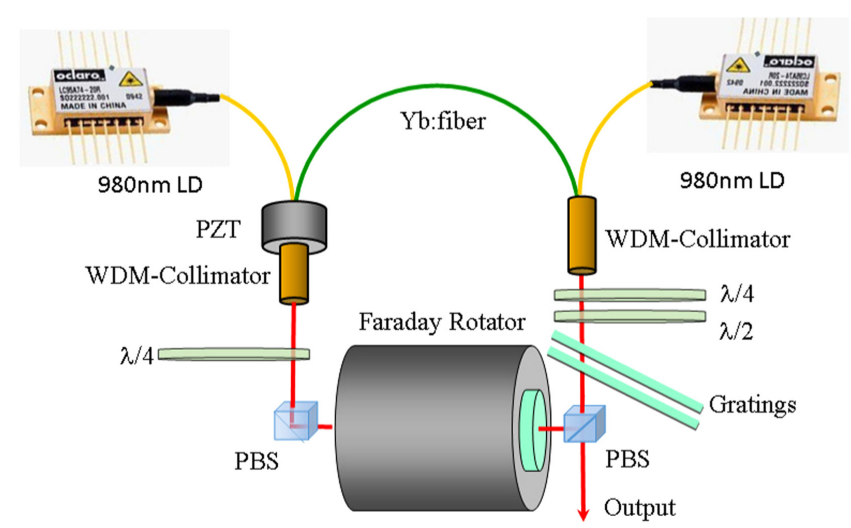

Figure 12. Configuration of $1 \mathrm{GHz}$ NPE mode locked fiber laser that delivers $60 \mathrm{fs}$ pulses at $>600 \mathrm{~mW}$ output power.

demonstrated with decent results, but the biggest challenge is that a fluoride fiber has a very different melting temperature from a silica fiber, which makes it very difficult to splice them together. It greatly limits the usage of fluoride fibers.

\section{Fiber optic beam delivery}

As mentioned in section 2, fiber delivery of high beam quality and high peak power pulses enables material and medical processes to be performed in confined areas. Solid-core fibers are unable to achieve this due to the dispersion and nonlinear optical effects.

\section{Advances in science and technology to meet challenges}

Novel saturable absorbers are still worth trying, including topological insulators [81]. Preference will be given to those with sub-ps recovery time, low saturation fluence, high modulation depth and high damage threshold.

The emerging of the biased NALM based 'figure 9' fiber laser (the broking of one of the rings in the figure ' 8 ' fiber laser makes the cavity like the number ' 9 ') [82] seems able to solve both self-starting and long-term reliability problems with unlimited lifetime. Such a technique is likely to become the overwhelming technique to replace most of the current mode locking mechanisms. The research topics left are how the cavity parameters affect the mode-locking threshold, output pulse duration and pulse energy, such as the beam splitting ratio, the amount of phase bias and the cavity configurations with extremely long or extremely short fiber length.

A rod type photonic crystal fiber is still the most capable fiber that offers the highest pulse energy among fiber lasers. Chirally coupled core ('3C') [83] and high order mode fibers were also proposed and demonstrated to generate tens of $\mathrm{nJ}$ pulse energy [84].

Combining a of bunch of fiber laser output into a pulse energy from $\mathrm{mJ}$ to $\mathrm{J}$ level is the ultimate way to increase pulse energy. The key technology is the coherent beam combining through the coherent amplification network [85], in which the seed pulse is split and amplified in separate fiber amplifier channels. The relative phases between different outputs are controlled so they can constructively produce a single higher power beam [86].

Alternatively, the combination with solid-state amplifiers in the final amplification stages, such as innoslabs [87], or zigzag slabs [88], may not be avoidable.

For fiber delivery, the hollow core Kagome fiber with negative curvature (figure 4, top) shows strong resistance to damage. The record of transmission peak power is $240 \mathrm{MW}$ with the intensity exceeding $10 \mathrm{TW} \mathrm{cm}^{-2}$ [89].

Multi-gigahertz rate fiber lasers rely on the progress of highly doped fibers. Although phosphite glass fibers can be heavily doped, they have the problem of splicing with silica fibers. Theoretical studies have paved the road for giant chirped pulse generation in super long fibers to mode lock in $<\mathrm{MHz}$ repetition rate.

Till now there are still no perfect solutions for MIR fiber lasers. Many researchers are devoted to improve the splicing technology by 'sticking' the fused silica fibers to fluoride fibers. A huge amount of effort has been invested in the development of fluoride fibers. With the advance of the industrial chain for fluoride fibers, more and more integrated components based on fluoride fibers will emerge, which would greatly expand their applications.

\section{Concluding remarks}

Ultrafast fiber lasers are becoming the fundamental elements of many optical systems for numerous industrial, medical and scientific applications. The applications can be as small-scale as ocular surgeries and biological imaging, or as large-scale as fiber communication and nuclear fusion; can be as precise as optical clock and molecular spectroscopy, or as brutal as laser machining and laser ignition of fusion. But at this point it is just the beginning of the era of ultrafast fiber lasers. There are still many challenges that lie ahead. The advance of novel fibers and mode-locking technology will stimulate more and more applications.

\section{Acknowledgments}

The author is grateful to Peng Li for the fruitful discussions and his contribution to part of the contents. 


\section{Microresonator sources of ultrafast pulses}

\section{Pascal Del'Haye}

National Physical Laboratory

\section{Status}

Optical microresonators have gained a lot of attention for nonlinear optics at extremely low threshold powers. The high optical quality factors combined with small mode volumes enables the observation of nonlinear optical effects like Raman scattering, Brillouin scattering, cavity optomechanics, parametric oscillations, and third harmonic generation at submilliwatt optical powers.

In recent years, optical microresonators have attracted significant interest for the generation of OFCs via four-wave mixing (microcombs) [63, 90, 91]. At present, microcomb generation has been observed in a variety of different materials, including fused silica, silicon, silicon nitride, magnesium fluoride, calcium fluoride, diamond, and aluminum nitride.

However, it only recently became possible to generate ultrafast pulses with whispering gallery mode microresonators for the first time. Here, it is important to realize that, unlike mode locked lasers, microresonator based FCs can emit a spectrum of equidistantly spaced frequencies that does not correspond to a pulse train [92]. Only a constant phase difference between all the comb modes leads to short pulses in the time domain. Generation of ultra-short pulses via soliton formation in microresonators has recently been observed in magnesium fluoride [93] and silicon nitride [94, 95] and fused silica resonators [99]. Moreover, recent advances in carrier envelope offset measurement [100] and stabilization [101, 102] could enable the generation of sub-cycle pulses directly within a microresonator.

Figure 13 shows the principle of microcomb generation via four-wave mixing and the difference to mode-locked lasers. Most importantly, a microresonator comb is generated directly from the pump laser via the $\chi^{(3)}$ nonlinearity of the resonator without an additional gain medium. This also implies that the pump laser is directly part of the comb spectrum of the microresonator, which enables direct control of the comb's center frequency and additional control of the carrier envelope offset frequency.

Reported pulse lengths from optical microresonators are around $200 \mathrm{fs}$ in magnesium fluoride and fused silica and silicon nitride resonators [99-101]. The shortest reported pulse length of $30 \mathrm{fs}$ has been reported in silicon nitride resonators at a repetition rate of $190 \mathrm{GHz}$ [93] (see figure 14).

Further reduction of the optical pulse length would make microresonator-based sources ideal tools for time domain ultrafast spectroscopy applications for out-of-the-lab use.

\section{Current and future challenges}

The main challenge for microresonator-based sources of ultrashort pulses is the compensation of microresonator dispersion
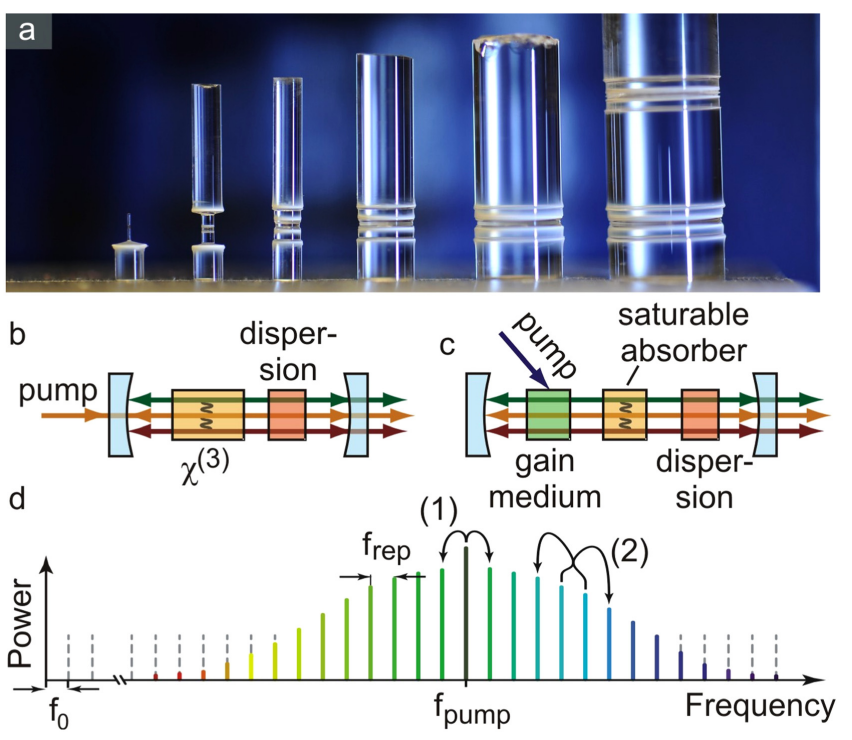

Figure 13. Optical frequency comb generation in microresonators. (a) Fused silica microrod resonators with diameters between $200 \mu \mathrm{m}$ and $8 \mathrm{~mm}$ (mode spacings from $300 \mathrm{GHz}$ to $8 \mathrm{GHz}$ ). Comb/pulse generation block diagram for microresonators (b) and mode locked lasers (c). Panel (d) shows the comb generation process in microresonators via degenerate (1) and non-degenerate four-wave mixing (2).

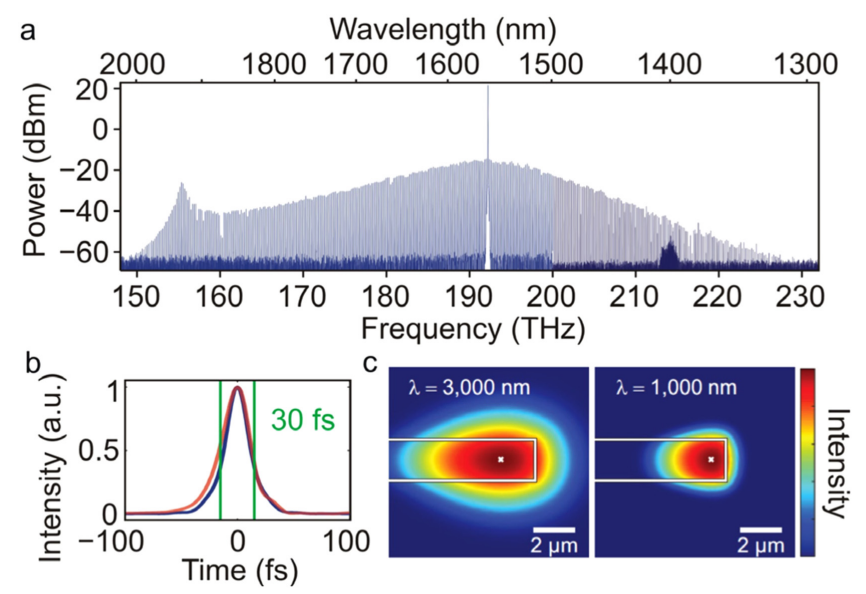

Figure 14. Panels (a) and (b) show the optical spectrum and calculated pulse width from a silicon nitride microresonator comb (data from Brasch et al [93]). Panel (c) shows the influence of geometric dispersion on the spatial position of the mode (from [103]). Precise control of the resonator geometry enables further reduction of the resonator dispersion with the goal of further spectral broadening of microcombs.

in order to increase the spectral bandwidth and reduce the pulse length. Conventional mode-locked lasers have the advantage that it is possible to precisely control the laser cavity dispersion with chirped mirrors, or prisms. In the case of microresonators, the dispersion has to be adjusted at the fabrication stage. However, several publications have pointed out ways to adjust dispersion by precise control of the geometry and material of the resonator [102-105]. Figure 14(c) shows the effect of the microresonator geometry on the spatial 
position of the modes, which contributes to the dispersion. However, dispersion compensation is even more complicated because the high power of the circulating pulse can significantly change the dispersive properties of the material. More research is required to overcome dispersion for further spectral broadening.

An alternative approach to intracavity spectral broadening is external broadening in a highly nonlinear fiber or a nonlinear integrated photonic waveguide. However, the big advantage of optical microresonators is the finesse of up to 1 million that leads to a huge power enhancement in the cavity, which would be very beneficial for spectral broadening in a dispersion engineered resonator.

An additional challenge of microresonators for ultrafast optics is the high repetition rate of the pulses. This repetition rate can be up to $1 \mathrm{THz}$ or higher. Importantly, a higher repetition rate corresponds to a smaller mode volume of the resonator, which is advantageous for the nonlinear comb generation process.

\section{Advances in science and technology to meet challenges}

In order to address dispersion compensation in optical microresonators it is necessary to invest more dedicated research in the optimization of resonator geometries. This can be achieved in different ways depending on the microresonator platform, for example, with additional cleanroom fabrication steps or precise laser machining. Additional requirements are tools and techniques for fast and reliable measurement of microresonator dispersion in order to achieve a fast turnaround in the resonator optimization process.

Regarding the high repetition rate of microcombs further development of electronics and photodetectors at $100 \mathrm{GHz}$ and beyond would help to achieve direct stabilization and comb control. This could be achieved using direct chip-integrated electronic feedback loops with a faster response time.

\section{Concluding remarks}

Microresonator sources for ultrafast optics have a high potential for precision time-domain spectroscopy in out-of-the lab applications. The most significant challenge is further intracavity spectral broadening of the emitted spectra. This could be achieved with a variety of different approaches including precision laser machining and novel multi-layer lithography techniques. High repetition rates and low power consumption make microresonator-based pulse sources ideal candidates for laser ranging and spectroscopy.

\section{Acknowledgments}

PD thanks Victor Brasch for providing the optical spectrum and pulse shown in figures 14(a) and (b). PD thanks the NPL Strategic Research Programme for providing funding for microcomb research. 


\section{Ultrafast solid-state laser sources}

\section{Thomas Südmeyer}

Université de Neuchâtel

\section{Status}

Extremely short pulse duration, high spatial coherence and broad optical spectrum - these unique properties of ultrashort laser pulses have resulted in numerous scientific breakthroughs. The short pulse duration allows resolving and even controlling processes that are extremely fast. Ultrashort laser pulses enabled Zewail to measure the dynamics of chemical reactions, a work honored by the 1999 Chemistry Nobel Prize (see section 5). The spectral properties of ultrafast lasers enable the generation of ultrastable OFCs (see sections 6 and 8), a work for which Hall and Hänsch received the 2005 Physics Nobel Prize. The high peak power allows efficient exploitation of nonlinear optical effects (see section 11) like e.g. in multi-photon imaging. Ultrafast lasers are an essential tool for many methods in super-resolved microscopy, an area for which Hell, Betzig and Moerner received the 2014 Nobel Prize in Chemistry. Intense ultrafast pulses even pave the way for novel extreme light-matter interactions, enabling table-top coherent X-ray sources (see section 5) and science at unprecedented short time scales of attoseconds. Initially, ultrafast lasers were nearly exclusively used in basic research. The complex, expensive and high-maintenance systems were not suitable for applications outside well-controlled lab conditions. The crucial task for bringing ultrafast lasers to an increasing number of wide-spread applications is reducing their complexity and price, while at the same time improving their performance and reliability. This became possible by ultrafast solid-state bulk and fiber lasers. In the following, we will focus on bulk lasers; a roadmap for fiber systems is presented in section 7. Key ingredients for this progress are the development of new solid-state gain materials [106], which combine efficient pumping by high power laser diodes with sufficient optical emission bandwidth for ultrashort pulse generation, the development of reliable pulse formation technologies like the SESAM [108], and the discovery of appropriate laser and amplifier designs for ultrashort pulses. Novel ultrafast diode-pumped solid-state lasers (DPSSLs) are currently entering more and more application fields in areas such as material processing, biology, chemistry, material science, and medicine. But there is still a large demand for further improvements in performance, cost, reliability and compactness, which is the target of many research teams and companies. Given the tremendous progress achieved during the last few years, I expect that the next generation of ultrafast laser sources will continue the tremendous progress in scientific discoveries and real-world use. Simpler, more compact, and more economical ultrafast solid-state lasers will enable new technologies and penetrate into numerous further application areas.

\section{Current and future challenges}

\section{Increasing the average power levels}

The development of ultrafast DPSSLs was the breakthrough for introducing ultrashort laser pulses in industrial production. Ultrashort pulses enable cold ablation: the pulse energy is absorbed via multiphoton processes in very thin layer. The direct transition from solid to gas prevents damage to the surrounding material and enables precise structuring in the micron or even nanometer range of virtually any material, even for extremely hard or inhomogeneous materials, which are difficult to process with other tools. Although this process was known for several decades, only recent ultrafast DPSSLs offer enough output power and stability to make their use cost-effective. Today, numerous laser companies offer picosecond DPSSLs with average power levels in the tens of watt range. A major challenge is the still relatively high production cost. Increasing the average power of reliable, cost-efficient and compact ultrafast laser sources into the hundreds or thousands of watt regime is an important target for operating at higher throughput in technical applications and for faster measurements in scientific experiments [108]. Moreover, many applications can benefit from shorter pulse durations in the femtosecond regime, and a second frontier is to extend the performance of high power ultrafast solid-state lasers towards shorter pulse durations. Areas such as structuring low-friction surfaces for efficient engines, efficient manufacturing of carbon fiber reinforced plastics, or generating customized microstructures for medical applications have a large growth potential.

\section{Expanding the spectral coverage}

Most commercially available ultrafast sources operate at a center wavelength in the range $800-1500 \mathrm{~nm}$. Ultrafast lasers operating at other wavelengths are highly attractive for expanding their application range. For example, ultrafast sources operating in the so-called 'fingerprint region' of 2-10 $\mu \mathrm{m}$, where many relevant molecules have characteristic fundamental absorption transitions, enable new sensing and spectroscopy methods [67]. In addition, longer wavelengths are promising for material processing of biomedical materials and plastics.

\section{Compact and reliable low-cost ultrafast sources for mass applications}

Although ultrafast lasers are used for production of mass devices, such as cell phones or parts in the automotive industry, they have not yet been integrated into devices sold in large quantities. Current ultrafast lasers typically cost more than 10k USD and are therefore only used in special devices with high value creation. Ultrafast lasers can improve mass applications such as telecommunication, range finding for the gaming industry, biomedical sensing and imaging, but the size, energy consumption and price is currently by far too high for these areas. 


\section{Advances in science and technology to meet challenges}

\section{Broad-band solid-state gain materials}

In order to achieve short pulse durations, gain materials with broad emission spectra are required. However, these materials usually have disordered crystal structure, which often restricts the propagation of phonons and leads to a significantly lowered thermal conductivity. One of the most mature gain material for ultrafast DPSSLs is Yb:YAG, which operates at a center wavelength of $1030 \mathrm{~nm}$, but only pulse durations of several hundred of femtoseconds in high power operation. The further research and growth optimization of spectrally broader gain materials with high thermal conductivity and mechanical strength that can be directly diode-pumped is of high importance, especially also operating in the mid-IR spectral region.

\section{Ultrafast thin disk and slab laser systems}

For high power ultrafast solid-state lasers, thermal lensing and aberrations in the gain media have to be overcome, which requires a laser design with excellent heat transport capabilities. Two very successful DPSSLs designs are the thindisk laser [109] and the slab design, because the ratio between cooling surface and active volume is large. The thin disk technology allows reaching extremely high output powers directly from oscillators without the need for further amplification [110]. Using amplifier approaches, both technologies have been achieving $\mathrm{kW}$-power levels in ultrashort pulses $[111,112]$. Further pushing the power levels by improved system designs, optimizing the repetition rates for the given applications, and reducing the pulse duration in high power operation are critical targets for the coming years.

\section{Optical components with extremely high damage thresholds}

Many high power femtosecond systems are currently not limited by the pump power or thermal effects in the gain, but by damage in other laser components. Investigating damage thresholds in the regime of multi-megahertz high power operation and optimizing components such as dispersive mirrors, pulse pickers, or modelocking elements is mandatory.

\section{New approaches for cost-efficient ultrafast lasers: waveguides,} green-diode pumped Ti:sapphire lasers, semiconductor disk lasers (SDLs)

Reducing the cost of ultrafast systems is another important step. For example, many multi-photon biomedical imaging systems still rely on Ti:sapphire lasers. Ti:sapphire lasers combine good thermal conductivity and extremely broad gain bandwidth, enabling the shortest achievable pulse duration directly from an oscillator, but require pumping in the bluegreen spectral region. Recently, green pump diodes have been developed for display applications, and they can be used for pumping Ti:sapphire at a small fraction of the usual costs. However, in the long term, replacing the existing ultrafast sources by a different technology with a higher level of integration appears mandatory to enable wide-spread applications. Ultrafast dielectric waveguide lasers are one promising approach for achieving this target. However, so far the achievable peak power level is still too low, and further research is required to increase it for real-world applications. Another promising approach is based on ultrafast SDLs [113]. Semiconductor lasers are a proven technology for cost-efficient mass production because they have a high level of integration. However, in standard edge-emitting devices, ultrashort pulses generate a large amount of nonlinear effects, which makes it very challenging to achieve high peak powers. This problem can be overcome by using vertical propagation through semiconductor layers, the so-called vertical external cavity surface emitting lasers or SDLs. Efficient ultrafast operation has been demonstrated and even integration of the pulse-forming semiconductor absorber into the gain chip has been shown in the MIXSEL (modelocked integrated externalcavity surface emitting laser, [114]). Important advances in this area are the combination of watt-level average powers with femtosecond operation, increasing the peak powers well above the $\mathrm{kW}$-regime, and further extension of the spectral operation range.

\section{Concluding remarks}

The future for ultrafast DPSSLs looks bright. Although the technology is already mature, the potential for research and paradigm-changing results is still very high. I expect that femotosecond lasers will expand into many additional research areas and industrial markets in the years ahead. For material processing and high field science, the average power levels of femtosecond systems will continue to increase into the kilowatt regime. On the other hand, opportunities for large-scale mass applications in areas like sensing, range finding, or imaging will open up by the development of compact and reliable low-cost femtosecond lasers based on new approaches such as ultrafast SDLs.

\section{Acknowledgments}

T Südmeyer acknowledges support by the Swiss National Science Foundation (SNF) (project 146738), the European Research Council (ERC Starting Grant 2011 \#279545), and Nano-Tera.ch MIXSEL II (20NA21_145932). 


\section{Compression of high energy pulses to the sub- attosecond regime: route to exawatt laser subatomic physics}

\author{
Gérard Mourou ${ }^{1}$ and Toshiki Tajima ${ }^{2}$ \\ ${ }^{1}$ École Polytechnique, Palaiseau \\ ${ }^{2}$ University of California at Irvine
}

\section{Status}

The possibility to amplify lasers to extreme peak power offers a new paradigm unifying the atomic and subatomic worlds, to include nuclear physics, high energy physics, astrophysics and cosmology. At the moment we are experiencing a rush toward the $10 \mathrm{PW}$ led by the 3-pillar ELI infrastructure along with Apollon in France and similar infrastructures in Russia, China and Korea. The applications include $\mathrm{x}$-ray and gammaray sources, high accelerating field gradients to the level of $\mathrm{GeV} \mathrm{cm}^{-1}$ with the goal to go beyond the high energy Standard Model and contribute to apprehend cosmic acceleration and revealing dark matter. The societal applications are also numerous with proton therapy, short-lived isotope production, nuclear waste transmutation and the like.

\section{Current and future challenges}

Over the past 30 years peak laser power has progressed to the petawatt $\left(10^{15} \mathrm{~W}\right)$ and is expected soon to reach $10 \mathrm{PW}$ (see figure 15). A shorter and cheaper route was recently presented utilizing existing modern PW level laser systems. Their peak power/intensity could be dramatically increased using today's energy level, i.e. 10-1000 J, by decreasing the pulse duration to the sub-attosecond time scale. This is possible using a two-step compression scheme [115]. The first relies on the flat top character in phase and amplitude of today's PW laser pulses. It makes possible to compress the pulse to the single cycle regime by producing a uniform self-phase modulation across the beam using a thin, sub-millimeter glass or plastic element at the laser output (see figure 16). The pulse could be subsequently compressed by a factor of 5-10 corresponding to pulse durations of $4-2 \mathrm{fs}$ by using an appropriate sequence of chirped mirrors. The resulting pulse of very large amplitude can be qualified using the normalized vector potential, $a_{0}=e E_{0} / m_{\mathrm{e}} \omega c$, which is $\sim 1$ when the laser intensity corresponds to $10^{18} \mathrm{~W} \mathrm{~cm}^{-2}$. When a laser pulse's $a_{0}$ reaches the range of 10-100 and is used to drive a relativistic plasma mirror. Naumova et al [116] modeled that in reflection a pulse duration as short as 600 attosecond $/ a_{0}$ with good $10 \%$ efficiency could be obtained using a single stage. Using two stages it should be possible to go to the subattosecond regime with an efficiency of $1 \%$. Note that a generated subattosecond pulse containing only $1 \mathrm{~J}$ will reach a peak power of the exawatt, and necessarily be in the keV-xray regime.

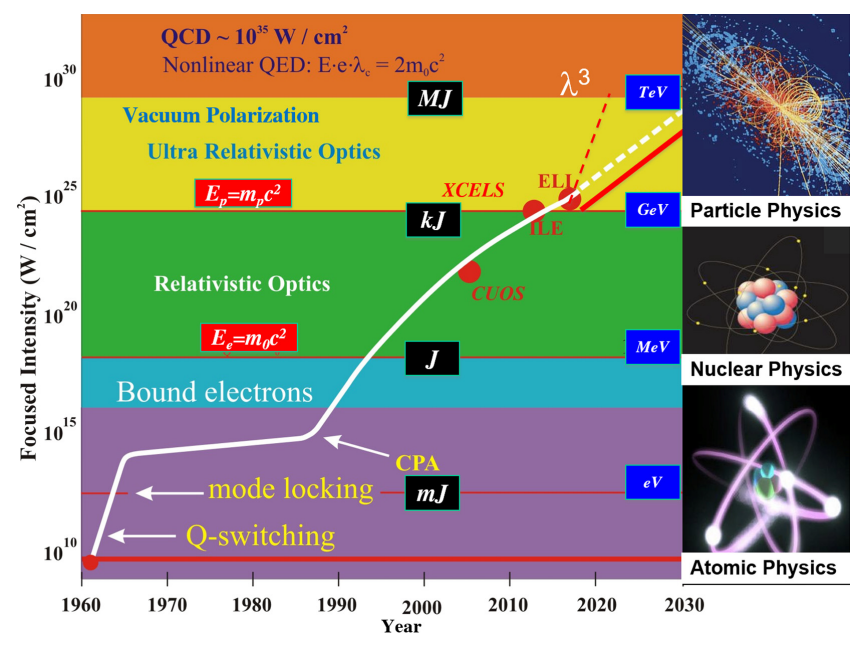

Figure 15. Laser Intensity through the years. The dashed line corresponds to what could be obtained with significant increases in beam size or by increasing the number of beams. The red-dashed line corresponds to the 'short cut' obtained through compression technique to sub-attosecond pulse durations. The plot has evolved from an early version first presented in [118].

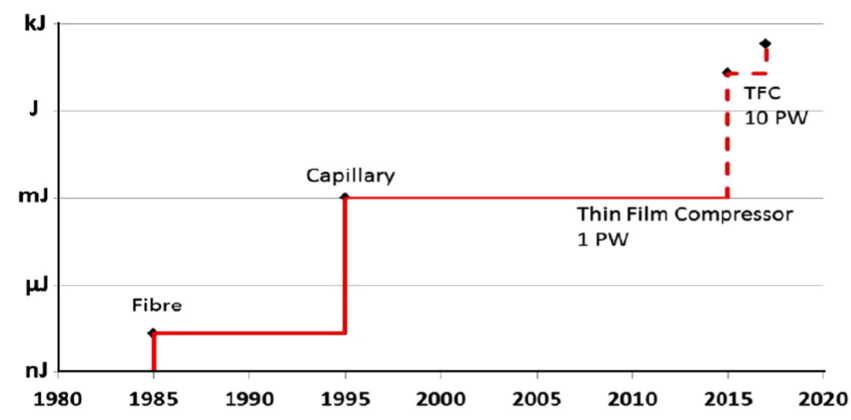

Figure 16. Evolution of pulse compression in the single cycle regime. Due to the energy losses associated with coupling the laser pulse spatial mode into a single-mode fiber or hollow-core capillary the resulting pulse energies from these schemes are typically limited to the sub $\mathrm{mJ}$ level. Proposed thin film compression techniques offer the possibility of compression laser pulses containing Joules of energy to durations of a few-cycles.

\section{Advances in science and technology to meet challenges}

The extraordinary large peak in the $\mathrm{keV}$-regime power will provide an accelerating gradient 6 orders of magnitude higher than what can be accomplished with RF based accelerators [117]. The critical density of a plasma, $n_{\mathrm{c}}$, is defined by a given laser frequency and increases with increasing photon frequency or energy. For $1 \mathrm{eV}$ optical photons, $n_{\mathrm{c}}$ is about $10^{21} \mathrm{cc}^{-1}$, while for $\mathrm{x}$-ray photons of $10 \mathrm{keV} n_{\mathrm{c}}$ is about $10^{27} \mathrm{cc}^{-1}$ and is well above solid densities. In laser wakefield acceleration (LWFA), the energy gain is limited by the critical density of the plasma with the high intensity LWFA energy gain given by

$\varepsilon_{\mathrm{e}}=a_{0}{ }^{2} m c^{2}\left(n_{\mathrm{c}} / n_{\mathrm{e}}\right)$, where $n_{\mathrm{e}}$ is the electron density. Therefore going to a higher photon energy allows for a greater achievable energy gain. An important benefit of a higher density plasma is that it supports a larger acceleration 
gradient. Shifting to extreme x-ray driven wakefield acceleration dramatically allows the acceleration to larger energies to be achieved over shorter distances and suggests $\mathrm{TeV}$ energies being achieved over centimeter distances. The accelerating lengths of the order of $\mathrm{TeV} \mathrm{cm}{ }^{-1}$ are $10^{3}$ times previous results achieved for optical lasers in gases and $10^{6}$ times that of current radiofrequency technology and are suggestive of accelerating to levels achieved only at CERN in Geneva, not over kilometers, but over a few centimeter focus - supported by a laser facility such as the PW level facilities currently being built.

\section{Concluding remarks}

Modern high peak power laser producing PW and $10 \mathrm{PW}$ pulses with top hat distribution, combined with a thin film/ plate compressor, could have the capability to produce $100 \mathrm{PW}$ pulses with single cycle or $2.5 \mathrm{fs}$, focused on a spot size limited by the laser wavelength. It is predicted that their interaction with solids will generate attosecond or even zeptosecond multi-exawatt pulses in the $\mathrm{x}$-ray regime with the acumen to drive giant acceleration in crystals in the $\mathrm{TeV} \mathrm{cm}{ }^{-1}$ regime. The combination of compression techniques forms the basis of an all-optical high energy physics field providing the means to investigate vacuum structure, dark matter, dark fields and the like. In addition the technology could underpin new compact sources of protons, neutrons and muons with applications in fundamental physics and societal applications like proton therapy or nuclear waste transmutation.

\section{Acknowledgments}

The authors wish to thank $\mathrm{N}$ Naumova and $\mathrm{J}$ Wheeler for their early work and discussions on the subject. 


\section{Nonlinear propagation phenomena in ultrafast optics}

\section{Daniele Faccio}

Heriot-Watt University

\section{Status}

One of the very first demonstrations of the capabilities of the newly invented LASER was second harmonic generation in a quartz crystal by Franken, in 1961. A series of studies quickly ensued and the foundations of nonlinear optics where already well established in the early sixties. In the 1970s, long distance guiding in optical fibers was also demonstrated and, following that, the realization that weak nonlinearity accumulated over long distances can lead to dramatic effects, ranging from soliton propagation to the generation of white light, also known as supercontinuum. A remarkable advance in this direction came with the invention of the photonic crystal fiber in the 1990s that enabled unprecedented control over laser pulse propagation and enhanced nonlinear effects. Nonlinear optics in photonic crystal fibers alone has developed into a whole research field. In conjunction with the demonstration of high precision FCs generated from mode-locked lasers for which a Nobel prize was awarded in 2005 (see sections 6 and 8), extremely high precision metrology with better than 1 part on $10^{17}$ is now achieved. Taking a step back in time again, possibly one of the most transformative changes in the field of nonlinear optics was the demonstration of lasing in titanium-doped sapphire materials and ultrashort laser pulse generation. Pulse durations reaching sub-100 fs are readily available and many labs around the world are currently working with $4 \mathrm{fs}$ durations (less that two optical cycles at $800 \mathrm{~nm}$ ).

In the late 1980s a remarkable observation opened yet another research area, 'ultrashort laser pulse filamentation': short, $100 \mathrm{fs}$ pulses left to propagate over long distances in air would self-focus to a small $\sim 100 \mu \mathrm{m}$ spot with remarkably high intensities in the $10 \mathrm{TW} \mathrm{cm}^{-2}$ region [119]. This focused pulse then propagated apparently without diffraction over many diffraction lengths, leaving a visible plasma filament in its wake. Ultrashort laser pulse filaments are currently used and studied for a variety of applications, ranging from pulse compression, white light generation, material processing and even weather control.

When optical pulses reach the few-cycle regime, new and unexpected effects emerge that can be grouped under the name of 'extreme nonlinear optics'. In many cases, the standard perturbative approach to nonlinear optics no longer holds and microscopic models that account either for the quantum properties and/or the non-power law dependence of light-matter interaction must be introduced. Possibly the most exciting example in this field is ' $\mathrm{HHG}$ ' that has been shown to lead to a plateau of harmonics extending all the way into the x-ray water window. When generated with single cycle pulses, the same process can generate a spectral continuum associated to an isolated pulse with durations demonstrated in the sub-100 as regime. It is worth noting that these are the shortest events of any kind made by man and are providing for example the first experimental insights into electron cloud dynamics.

\section{Current and future challenges}

One of the greatest challenges and avenues for future research is related to the fact that Ti:sapphire technology is so well established that researchers currently have easy and commercial access to robust femtosecond pulse light sources. On the one hand this has enabled most if not all of the more remarkable feats of nonlinear optics but on the other has limited our view of light - matter interaction to a rather restricted spectral bandwidth centered in the near-infrared region, below $1 \mu \mathrm{m}$. Results accumulated over the past few years have already very clearly shown that extending the wavelengths towards the UV, MIR or even far-infrared (FIR) is not simply a question of 'more of the same' but truly new propagation regimes are observed. Examples are unexpected phasematching conditions for HHG in the x-ray water window region when using few cycle MIR pulses [120] or enhanced THz (FIR) generation with MIR pumped plasma filaments [121].

A large obstacle to be overcome here is the availability of high power, few-cycle MIR pulsed laser sources. Current approaches involve either optical parametric amplification (OPA) (therefore, enabled by nonlinear optics) or novel laser materials with large gain bandwidth. However, the former is not readily scalable to high intensities (as it in turn requires even high power pump lasers) and the latter does not have access, yet, to a wide range of materials (although the lesson learnt from the history of the Ti:sapphire laser tells us that only one suitable material is required in order to revolutionize a research field).

There seems to be emerging evidence that it is not only HHG or THz generation that is strongly affected by the use of MIR pump pulses but, in general, most fields of high intensity nonlinear optics. The reasoning behind this is that the interactions are now occurring in a regime where, in most materials, dispersion becomes a secondary effect or, stated differently, the dynamics are dominated by the short nonlinear length. One example is filamentation where preliminary studies have already shown that MIR pulses lead to peculiar propagation features. One outstanding goal for filamentation that will be required if filaments are to deliver on some of the promised applications is the extension of the filament length beyond the current few meter lengths, combined also with a suppression of multiple filament breakup and/or concentration of higher energies into a single filament. These in turn would have an impact on some of the more challenging proposals for filamentation optics, such as lightning (or high energy voltage discharge) guiding or rain/snowfall control [122].

Changing topic slightly, fiber-based nonlinear optics is still reserving surprises and potential for future fundamental studies and applications alike. Examples are the recent demonstrations of very strong nonlinear dynamics in multimode fibers [123] and the observation of soliton interaction in fiber cavities over distances that are measured in astronomical units [124]. The latter therefore enable the study of extremely weak effects, corresponding to temporal delay accumulations of the order of 1 as per cavity round trip. 
Nonlinear interactions in the 'weak' regime are in general of interest for a number of applications, one of the most obvious being single photon switches for quantum computing. One example is nonlinear optics with high-level energy excited Rydberg atoms that exhibit a remarkably strong interaction with light due to the large micron-scale diameters and huge geometric cross-section. These allow efficient light-matter interactions at the single photon level. 'Quantum nonlinearity', in the wider sense of nonlinear interactions, with specifically engineered photon states is a very promising future direction. It has been shown for example that non-classical light from a broadband down-conversion process will behave as a single photon in a two-photon process (which therefore shows a linear growth in intensity instead of the quadratic growth observed with classical light states) [125]. It could in principle be possible to vastly increase nonlinear interaction efficiencies also in other processes, e.g. harmonic or even HHG using properly chosen photon states. The main challenge here remains the creation of a bright light source that can deliver the desired, e.g., high-number Fock states.

Another growing field of interest in nonlinear optics is the possibility to use light to mimic other systems. The most wellknown example is the use of light propagation in optical fibers or other systems in regimes that exhibit rare, yet extreme-height events. The first note on the importance of these events was based on soliton fission in a fiber, exhibiting rare but very intense Raman red-shifting of solitons [126]. These dynamics were likened and compared to oceanic rogue waves. There is now a consistent literature on the topic of optical rogue events: the field has opened a new understanding in optical soliton propagation dynamics but arguably has also had a strong impact on the oceanic studies where, until recently, rogue waves were not even seriously considered due to the lack of systematic measurements. Oceanic rogue waves are currently the object of intense scrutiny and studies that are at least partly based on the understanding and methods emerging from the nonlinear optics community. In a similar fashion, there are other apparently unrelated fields that can be connected to nonlinear optical propagation. Going beyond the rogue wave analogy, it is possible to create actual fluids made of light [127]. Repulsive photon-photon interactions arising from a negative
Kerr-like nonlinearity lead to an effective superfluid-like evolution of the light beam, which can be used to observe dynamics that are typically associated to superfluid Helium or Bose-Einstein condensates. These photon fluids can also be used to mimic curved spacetime geometries, providing a remarkable connection to general relativity and gravitational physics. Similar ideas, such as the creation of artificial event horizons and even Hawking radiation emission from these have been proposed in solitons and intense light pulses [128]. The concept of an optical horizon has gradually emerged over the past years in nonlinear optical fiber propagation and has been proposed for a range of applications such as coherent supercontinuum generation, wavelength control and all-optical transistors. One of the open challenges is to transform these gravitational analogues into systems that can provide valuable information for those working with the Einstein equations and relative astrophysical or cosmological models, possibly even looking as far as quantum gravity predictions.

\section{Concluding remarks}

We have attempted to give a brief overview of some of the major achievements of propagative nonlinear optics together with some outstanding challenges and potential avenues for future research. This overview is of course biased by personal preference and there are certainly many other opportunities in the field.

But regardless of the actual applications involved, the parabola of the progress from the first Ruby laser to attosecond, and possibly in the future even zeptosecond pulses is constellated with a remarkable variety of achievements and is a testament to mankind's ingenuity, driven by curiosity, the desire to understand nature and, of course, nonlinear optics.

\section{Acknowledgments}

DF acknowledges support from the European Research Council under the European Union's Seventh Framework Programme (FP/2007-2013)/ERC GA 306559. 


\section{Sensing and spectroscopy}

\section{Frans J M Harren}

Radboud University

\section{Status}

Since the invention of the laser, spectroscopy made a significant step forward in helping to understand the properties of materials in the gas, liquid or solid phase. Before this invention Globar sources were used, in combination with interferometric methods, to study electronic, vibrational and rotational transitions covering the UV, visible, near- and MIR wavelength region. Due to their monochromatic properties lasers are able to sense molecules with high sensitivity and selectivity, taking advantage of their high spectral resolution and the ability to operate over long optical path lengths. The first laser-based spectroscopic sensing can be found in the early 1970s when nitric oxide (NO) was detected as pollutant in air samples [129]. In that time period the laser was widely accepted as a new high resolution spectroscopy tool. Lasers were developed in two directions: as pulsed or as continuous wave source. Pulsed lasers have high intensity, short pulses starting from Q-switching (nanosecond scale) towards modelocked systems (femtosecond scale). The advantage of pulsed systems is that they can initiate multi-photon excitation and electronically excite, ionize and dissociate molecules; besides intermediating chemical reactions. Their downside was their pulse to pulse fluctuations and varying pulse shapes, inducing strongly fluctuating nonlinear effects, making them less convenient for sensing applications in which high sensitivity and precision is needed. Sensing with continuous wave lasers became the standard for spectroscopic sensing applications (see e.g. [130]), due to their narrow linewidth and stable output power. For many spectroscopic sensing applications a distributed feedback laser with a limited tuning range is sufficient. It will keep costs low, important for end users within industrial process monitoring, atmospheric chemistry, but also medical sciences and agrotechnology applications [131]. However, in many applications sensing gets impeded by spectroscopic interferences of other molecular species or a fringing effect of the optical system itself. Especially, investigating complex mixtures demands for a wide wavelength coverage with widely tunable lasers and nonlinear devices (such as external cavity QCLs, OPOs or difference frequency generators). Not to exceed a reasonable measuring time such devices should be able to scan quickly and still sense properly.

\section{Current and future challenges}

Spectroscopic sensing is important in many aspects of science and society (see illustration in figure 17); one of the most important spectroscopic instruments which is used for this is FTS. The FTS is the most efficient approach to record highly consistent, well resolved spectra over a wide wavelength coverage, ranging from the infrared to VUV. They have a position of dominance in the field of spectroscopy as the versatility of this technique and the relatively low cost of the instrumentation proved much appropriate for the determination of the structure of molecules, the quantitative analysis of complex mixtures, the investigation of dynamic systems, biomedical spectroscopy, hyperspectral imaging, and the study of many types of interfacial phenomena. Despite its success, the FTS has its limitations. Recording times are limited by the speed of a moving mirror and often exceeds several minutes, which is orders of magnitude too long for applications linked to real-time monitoring. Due to the use of low-brightness incoherent light sources, FTS exhibits limited sensitivity with a limited optical path length.

Here lies a challenge for ultrafast optics, and especially FC spectroscopy, to renew the field of FTS towards high resolution, broadband spectroscopy under dynamically changing conditions with a high sensitivity. More specifically, the following conditions have to be met:

- There is a need for a high sensitivity, which not only includes the development of the optical source but also a long optical interaction length, a sensitive and selective detection system and a proper data handling.

- Sufficient large spectral bandwidth; such that complex mixtures can be observed or a global wide observation of different energy levels in one specie.

- High spectral resolution, especially for gas phase spectroscopy at sub-Doppler resolution. It will give selectivity against other species and isotopologues.

- Fast acquisition time such that short-lived phenomena can be observed performing time-resolved studies.

- Frequency accuracy, a self-calibrated wavelength scale will obsolete the need of a wavemeter or spectrum analyzer.

Continuous wave, tunable lasers can address these challenges using appropriate spectroscopic detection schemes, but as soon as broad spectral coverage is needed at seconds' timescale they become inefficient. As a consequence, in recent years, one of the major preoccupations of spectroscopists is to elaborate new strategies for the next generation of spectroscopic instruments able to deliver at once high resolution, high accuracy, broad spectral coverage and rapid acquisition.

\section{Advances in science and technology to meet challenges}

Since 2000, the FC [132] has appeared as a new laser source offering broad spectral extension and high brightness. The FC is typically based on femtosecond modelocked lasers, but can also be generated via micro resonators [90] or QCLs [133]. Its spectral coverage (typically several hundreds of wavenumbers) can be extended via nonlinear generation (supercontinuum, difference frequency, optical parametric generation) [67]. Its spectral structure is made of numerous equidistant narrow lines that have stable and accurate individual positions. They can be described as a bright source 


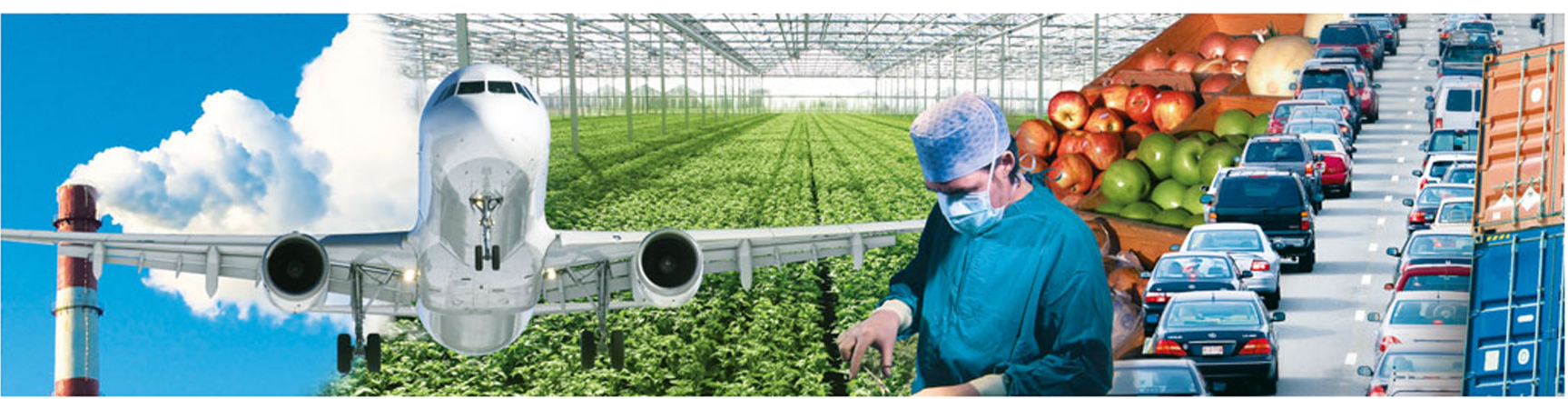

Figure 17. An illustration of the importance of sensing in industrial, medical and scientific applications, as well as society.
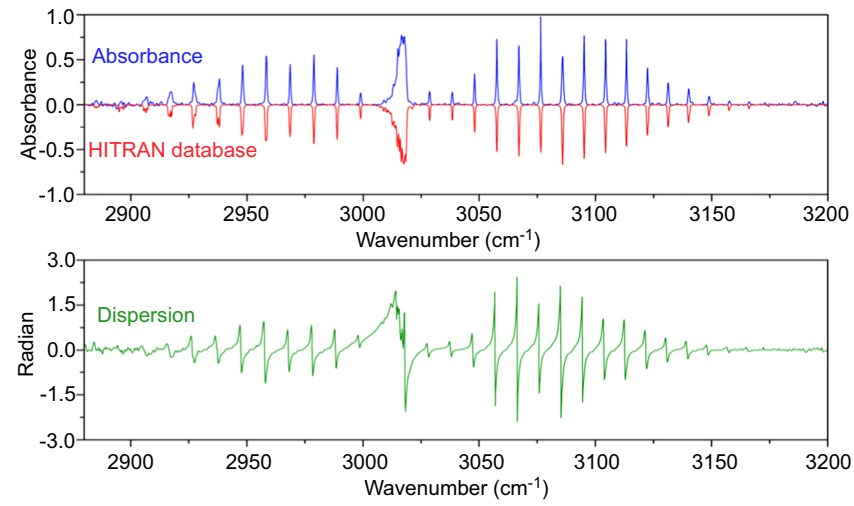

Figure 18. Spectroscopic example using a frequency comb Fourier transform spectrometer showing the $\nu_{3}$-vibrational band of methane with a wavelength coverage of $350 \mathrm{~cm}^{-1}$, a spectral resolution of $0.3 \mathrm{~cm}^{-1}$ within a recording time of $100 \mathrm{~ms}$. A comparison is made with the HITRAN database; for more details see [136].

equivalent to $100000 \mathrm{~s}$ single-mode lasers emitting at perfectly well known frequencies. As such, FCs are presently transforming the scientific field within fundamental metrology and spectroscopy. For spectroscopic analysis, FC lasers were successfully combined with Michelson-based FTS (see e.g. [134]) and the outbreak of FC lasers has led to a new generation of spectrometers, called the FC-FTS or dual-comb spectrometer, in which two FC lasers are used for spectroscopy [135]. The operation principle of the FC-FTS is that the beat notes between pairs of lines from two combs with different repetition frequencies occur in the RF domain, thus providing instantaneously a down-converted image of the optical spectrum. See figure 18 for an example spectrum. Due to the brightness of laser FCs, long averages are not needed to obtain high signal-to-noise ratios, and broadband and resolved molecular spectra are recorded within tens of milliseconds.

FC-FTS has great potential to reach high sensitivity, as it takes advantages of the brightness of the lasers sources. The signal is detected at RF frequencies, allowing shot-noise-limited detection. However, it remains a challenge to generate stable MIR FCs with the wavelength coverage of a Globar source $(2-20 \mu \mathrm{m})$ and enough optical power. The MIR wavelength region is important as this is the molecular fingerprint region, in which molecules can be identified with high selectivity and sensitivity. Recently, on a number of occasions, sensitive spectroscopy using Dual Comb FTS has been demonstrated in the near and mid infrared wavelength regions.

\section{Concluding remarks}

There is a challenge for FC spectroscopy to renew the field of FTS towards high resolution, broadband spectroscopy under dynamically changing conditions with a high sensitivity. Speed, wavelength coverage and spectral resolution are the main opportunities to establish a presence in the field of spectroscopic sensing. To develop instrumentation outside the $\mathrm{R} \& \mathrm{D}$ field, other factors such as price and size will become important factors. This can only be overcome by miniaturization and photonic-electronic integration. For the nearinfrared wavelength region major steps are already being made in this direction, developing integrated dual comb modelocked lasers.

\section{Acknowledgments}

The author acknowledges the expert advice and helpful discussions with J Mandon. 


\section{Parametric frequency conversion}

\section{Giulio Cerullo}

Politecnico di Milano

\section{Status}

State-of-the-art femtosecond lasers, based on Ti:sapphire or Yb:doped gain media, operate at fixed wavelengths $(0.8 \mu \mathrm{m}$ for Ti:sapphire and $1 \mu \mathrm{m}$ for $\mathrm{Yb})$. This contrasts with the requirements of many applications, which call for ultrashort light pulses broadly tunable from the MIR to the ultraviolet. As there are no classical laser active media capable of providing gain over such a broad frequency range, frequency tunability must be achieved by a nonlinear optical effect, taking advantage of the high peak powers of femtosecond lasers. The most widespread solution exploits the second order nonlinear effect known as OPA [137]. In an OPA (figure 19(a)) energy is transferred, in a nonlinear crystal, from an high frequency and high intensity beam (the pump, at frequency $\omega_{\mathrm{p}}$ ) to a lower frequency, lower intensity beam (the signal, at frequency $\omega_{\mathrm{s}}$ ) which is thus amplified, starting from a weak 'seed' beam; in addition a third beam (the idler, at the difference frequency $\omega_{\mathrm{i}}=$ $\left.\omega_{\mathrm{p}}-\omega_{\mathrm{s}}\right)$ is generated. The OPA process satisfies energy conservation and requires, for efficient energy conversion, momentum conservation (phase-matching) $k_{\mathrm{p}}=k_{\mathrm{s}}+k_{\mathrm{i}}$. The OPA is thus an optical amplifier with continuously variable center frequency (determined by the phasematching condition) and represents an easy way of tuning over a broad range the frequency of an otherwise fixed femtosecond laser system [138]. On the other hand, if suitably designed, an OPA can simultaneously fulfill the phase-matching condition for a broad range of signal frequencies thus acting as a broadband amplifier, efficiently transferring energy from a narrowband pump pulse to a broadband signal (idler) pulse; it can therefore be used to dramatically shorten, by over an order of magnitude, the duration of the pump pulse, generating tunable few-opticalcycle pulses (figure 20) [139]. Femtosecond OPAs, using Ti:sapphire pump lasers and BBO nonlinear crystals, are a rather mature technology, with well-understood design principles and a broad range of applications, from ultrafast optical spectroscopy (see section 5) to high-field science (see section 1). The tunability of OPAs can be extended in the UV and the IR spectral ranges by other second-order nonlinear processes, such as sum- and difference-frequency generation.

\section{Current and future challenges}

There is a huge development potential for Yb:pumped OPAs. $\mathrm{Yb}$ lasers can be dramatically scaled (see section 9) in both average power (up to several $\mathrm{kWs}$ ) and repetition rate (up to the $\mathrm{MHz}$ range), but have narrow gain bandwidths. (a) - OPA

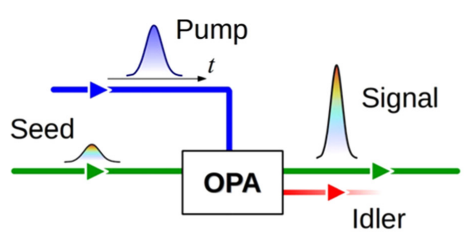

(b) - OPCPA

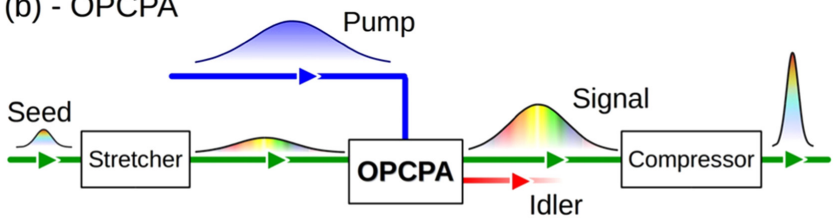

Figure 19. (a) Conceptual scheme of an ultrafast OPA; (b) conceptual scheme of an OPCPA.

Broadband Yb-pumped OPAs allow the generation of fewoptical-cycle pulses at high average powers and repetition rates, for many applications which benefit from high photon flux [140].

Optical parametric chirped-pulse amplification (OPCPA) [129] consists in amplification of a chirped seed pulse by a long (1-10 ps) pump pulse, followed by recompression (figure 19(b)). With respect to conventional chirped-pulse amplification it has several advantages: (i) capability of providing high gain in a short pathlength; (ii) ultrabroad gain bandwidths; (iii) reduction of pre-pulse pedestals due to amplified spontaneous emission; (iv) low thermal loading due to lack of energy storage. OPCPA is the most promising route for power scaling of few-optical-cycle pulses, up to the PW level [142] (see section 10).

OPAs enable passive, all-optical stabilization of the carrier-envelope phase (CEP) of ultrashort pulses. This effect exploits the cancellation of the CEPs of the interacting beams in the DFG process which is intrinsic to OPAs [143]. Passive CEP stabilization has advantages with respect to active approaches, such as the absence of electronic circuitry, frequency tunability and the possibility of combining ultrabroad bandwidths with energy scaling, using OPCPAs.

Broadband OPAs have been optimized, at different frequencies, in terms of amplified bandwidth, resulting in the shortest possible pulsewidths. A further bandwidth increase can be obtained by coherent synthesis of pulses with different colors, generated by separate OPAs. In combination with CEP control, coherent synthesis will allow to shorten the pulsewidth to the sub-cycle limit and generate high-power light transients with controlled electric fields, allowing on-demand optical waveform generation [144].

Another challenge is the generation of powerful fewoptical-cycle pulses in the MIR range, which, due to the $\lambda^{2}$ scaling of the ponderomotive energy of free electrons, will lead to enhanced strong-field light-matter interactions such as HHG [145] (see section 1). Extension of frequency tunability to the UV range by up-conversion will also be of great interest for studies on biomolecules (DNA, proteins). 
Frequency $(\mathrm{THz})$

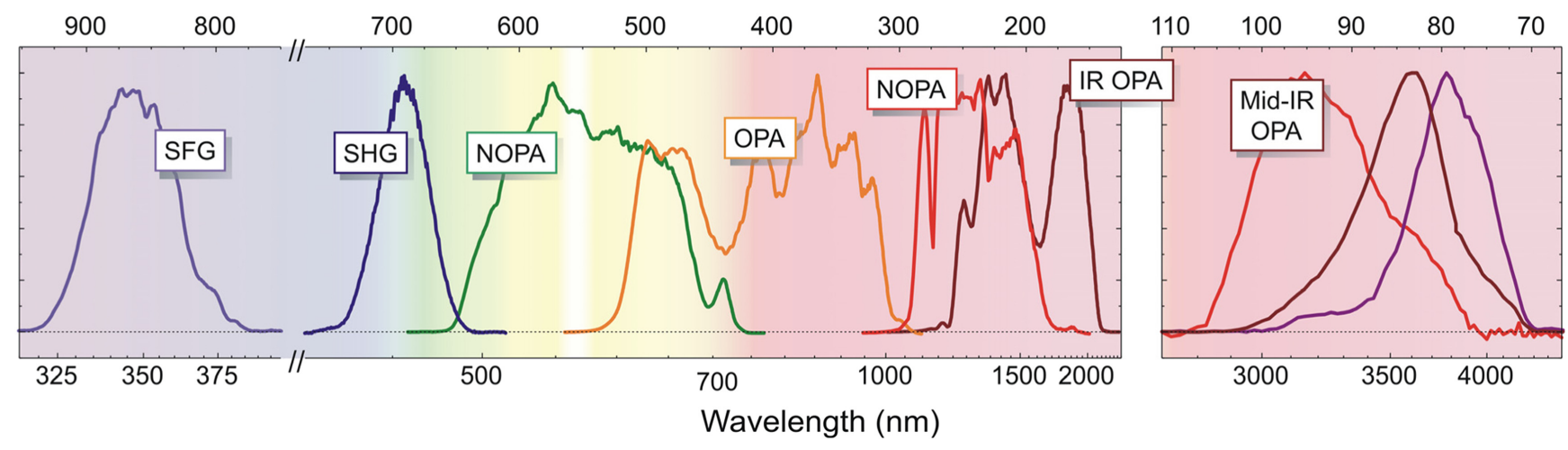

Figure 20. Examples of tunable few-optical-cycle pulses generated by OPAs under broadband phase matching conditions, and spanning a wide spectral range [139]. The UV pulses are generated by frequency up-conversion. NOPA: non-collinear OPA; SHG: second-harmonic generation; SFG: sum-frequency generation.

\section{Advances in science and technology to meet challenges}

Scaling femtosecond OPAs to high average powers and high repetition rates will require the development of adequate pump lasers, based on $\mathrm{Yb}$ technology, either in bulk format (using thin disk or slab geometries or cryogenic cooling of the active medium) or in fiber format (with coherent beam combination from parallel amplifiers, see section 7). At such average powers one should consider thermal effects in the OPA crystals, due to parasitic linear or multiphoton absorption, which may lead to beam distortions, deviations from the phase-matching conditions and catastrophic failure.

The development of OPCPA technology and its peak power and pulse energy scaling capabilities will depend on the availability of suitable pump laser systems, with pulse duration of a few picoseconds, which are challenging to generate at high energies and/or repetition rates. The spatial and temporal beam quality of such pump lasers will have to be optimized, as it directly impacts on the OPCPA performance, and parasitic parametric superfluorescence will have to be suppressed.

Coherent synthesis of multiple $\mathrm{OP}(\mathrm{CP}) \mathrm{A}$ channels promises to deliver optical waveforms with multi-octave spectra and sub-cycle control of the electric field, opening the field of 'waveform nonlinear optics'. This development poses formidable challenges: dispersion management has to be implemented over ultrabroad bandwidths, using specially developed chirped mirrors and adaptive optics. In addition the timing between the different $\mathrm{OP}(\mathrm{CP}) \mathrm{A}$ channels has to be controlled with sub-100 attosecond accuracy, using balanced optical cross-correlator technology.

Passive CEP stabilization needs to be further investigated and its performances, in terms of CEP fluctuations, assessed against those of the more established active approaches.

MIR pulses are currently produced by DFG between the signal and idler pulses of an OPA, with limited energy and bandwidth. Current pump sources, at 0.8 and $1 \mu \mathrm{m}$, do not allow to generate mid-IR pulses directly as the idler of an OPA, due to two-photon pump absorption in the OPA crystal (s). The ongoing development of powerful sources of ultrashort pulses at $\approx 2 \mu \mathrm{m}$, based on Ho: and/or Tm:doped gain media [145], will allow direct pumping of mid-IR OPAs based on $\mathrm{ZnGeP}_{2}$, GaSe and $\mathrm{AgGaSe}_{2}$ crystals with lower quantum defects and more favorable group-velocity matching.

\section{Concluding remarks}

Parametric frequency conversion, mainly based on OPAs, is a mature technology enabling femtosecond lasers, which operate at fixed wavelengths, to achieve tunability over the whole electromagnetic spectrum, from the MIR to the ultraviolet. This gives an unprecedented flexibility to studies of light-matter interaction, both in the perturbative and the strong-field regimes. In addition, thanks to their broad gain bandwidths, OPAs have the unique capability to generate tunable few-optical-cycle light pulses. OPA technology can be scaled to very high average powers (thanks to the development of Yb lasers) and peak powers (thanks to the OPCPA concept). Future challenges will concern: control of the CEP of the generated pulses, by passive stabilization mechanism; further bandwidth broadening, to the multi-octave regime, by coherent synthesis of OPAs; extension of few-optical-cycle pulse generation to the MIR and ultraviolet ranges, which are critical for many applications.

\section{Acknowledgments}

The author acknowledges useful discussions with Dr Cristian Manzoni. 


\section{References}

[1] Spence D E, Kean P N and Sibbett W 1990 Sub-100 fs pulse generation from a self-modelocked titanium: sapphire laser Conf. on Lasers and Electro-optics, CLEO pp 619-20

[2] Hentschel M et al 2004 Science 414509

[3] Leone $\mathrm{S}$ et al 2014 What will it take to observe processes in 'real time'? Nat. Photon. 8162

[4] Krausz F and Stockman M 2014 Attosecond metrology: from electron capture to future signal processing Nat. Photon. 8205

[5] Vampa G, Hammond T J, Thiré N, Schmidt B E, Légaré F, McDonald C R, Brabec T and Corkum P B 2015 Linking high harmonics from gases and solids Nature $\mathbf{5 2 2} 462$

[6] Calegari F et al 2014 Ultrafast electron dynamics in phenylalanine initiated by attosecond pulses Science 346336

[7] Miao J, Ishikawa T, Robinson I K and Murnane M M 2015 Beyond crystallography: Diffractive imaging using coherent x-ray light sources Science $\mathbf{3 4 8} 530$

[8] Cingöz A, Yost D C, Allison T K, Ruehl A, Fermann M E, Hartl I and Ye J 2012 Direct frequency comb spectroscopy in the extreme ultraviolet Nature $\mathbf{4 8 2} 68$

[9] Louisy M et al 2015 Gating attosecond pulses in a noncollinear geometry Optica 2563

[10] Wirth A et al 2011 Synthesized light transients Science 334195

[11] Heyl C M et al 2016 Scale-invariant nonlinear optics in gases Optica 375

[12] Silva F, Teichmann S M, Cousin S L, Hemmer M and Biegert J 2015 Spatiotemporal isolation of attosecond soft X-ray pulses in the water window Nat. Commun. 66611

[13] Hernandez-Garcia C et al 2013 Zeptosecond high harmonic $\mathrm{keV} \mathrm{x}$-ray waveforms driven by midinfrared laser pulses Phys. Rev. Lett. 111033002

[14] Takahashi et al 2002 Generation of $10-\mu \mathrm{J}$ coherent extremeultraviolet light by use of high-order harmonics Opt. Lett. 271920

[15] Rudawski et al 2013 A high-flux high-order harmonic source Rev. Sci. Instrum. 84073103

[16] Constant et al 1999 Optimizing high harmonic generation in absorbing gases: model and experiment Phys. Rev. Lett. 821668

[17] Hädrich et al 2014 High photon flux table-top coherent extreme-ultraviolet source Nat. Photon. 8779

[18] Lorek et al 2014 High-order harmonic generation using a high-repetition-rate turnkey laser Rev. Sci. Instrum. $\mathbf{8 5}$ 123106

[19] Cabasse et al 2012 Optimization and phase matching of fiberlaser-driven high-order harmonic generation at high repetition rate Opt. Lett. 374618

[20] Hädrich et al 2015 Exploring new avenues in high repetition rate table-top coherent extreme ultraviolet sources Light Sci. Appl. 4 e 320

[21] Heyl et al 2012 High-order harmonic generation with $\mu \mathrm{J}$ laser pulses at high repetition rates J. Phys. B 45074020

[22] Lindner et al 2003 High-order harmonic generation at a repetition rate of $100 \mathrm{kHz}$ Phys. Rev. A $68013814 / 1$

[23] Gattass R R and Mazur E 2008 Femtosecond laser micromachining in transparent materials Nat. Photon. 2219

[24] Rekow M et al 2014 Precision glass processing with picosecond laser pulses Industrial Laser Solutions for Manufacturing

[25] Tanous A C 2010 Laser cutting takes the heat out of stent manufacturing Industrial Laser Solutions for Manufacturing

[26] Toenshoff H K, Ostendorf A, Nolte S, Korte F and Bauer T 2000 Micromachining using femtosecond lasers Proc. SPIE 4088136
[27] Lubatschowski H, Maatz G, Heisterkamp A, Hetzel U, Drommer W, Welling H and Ertmer W 2000 Application of ultrashort laser pulses for intrastro- mal refractive surgery Graefes Arch. Clin. Exp. Ophthalmol. 23833

[28] Thomson R R, Bookey H T, Psaila N D, Fender A, Campbell S, MacPherson W N, Barton J S, Reid D T and Kar A K 2007 Ultrafast-laser inscription of a three dimensional fan-out device for multicore fiber coupling applications Opt. Express 1511691

[29] Thomson R R, Birks T A, Leon-Saval S G, Kar A K and Bland-Hawthorn J 2011 Ultrafast laser inscription of an integrated photonic lantern Opt. Express 195698

[30] Salter P S and Booth M J 2011 Addressable microlens array for parallel laser microfabrication Opt. Lett. 362302

[31] Pospiech M, Emons M, Väckenstedt B, Palmer G and Morgner U 2010 Single-sweep laser writing of 3Dwaveguide devices Opt. Express 186994

[32] Jaworski P, Yu F, Carter R M, Knight J C, Shephard J D and Hand D P 2015 High energy green nanosecond and picosecond pulse delivery through a negative curng $O p t$. Express 238498

[33] Wong T C, Rhodes M and Trebino R 2014 Single-shot measurement of the complete temporal intensity and phase of supercontinuum Optica 1 119-24

[34] Bowlan P, Fuchs U, Trebino R and Zeitner U D 2008 Measuring the spatiotemporal electric field of tightly focused ultrashort pulses with sub-micron spatial resolution $O p t$. Express 16 13663-75

[35] Fisher R A and Fleck J J A 1969 On the phase characteristics and compression of picosecond pulses Appl. Phys. Lett. 15 287-90

[36] Trebino R 2002 Frequency-Resolved Optical Gating: The Measurement of Ultrashort Laser Pulses (Dordrecht: Kluwer)

[37] Rhodes M, Steinmeyer G, Ratner J and Trebino R 2013 Pulse-shape instabilities and their measurement Laser Photon. Rev. 7 557-65

[38] Quere F, Mairesse Y and IItatani J 2005 Temporal characterization of attosecond XUV fields J. Mod. Opt. 52 339-60

[39] Akturk S, D’Amico C and Mysyrowicz A 2008 Measuring ultrashort pulses in the single-cycle regime using frequencyresolved optical gating J. Opt. Soc. Am. B 25 A63-69

[40] Rhodes M, Steinmeyer G and Trebino R 2014 Standards for ultrashort-laser-pulse-measurement techniques and their consideration for self-referenced spectral interferometry Appl. Opt. 53 D1-11

[41] Extermann J, Bonacina L, Courvoisier F, Kiselev D, Mugnier Y, Le Dantec R, Galez C and Wolf J-P 2008 NanoFROG: frequency resolved optical gating by a nanometric object Opt. Express 16 10405-11

[42] Bowlan P and Trebino R 2012 Using phase diversity for the measurement of the complete spatiotemporal electric field of ultrashort laser pulses J. Opt. Soc. Am. B $29244-8$

[43] Guang Z, Rhodes M, Davis M and Trebino R 2014 Complete characterization of a spatiotemporally complex pulse by an improved single-frame pulse-measurement technique $J$. Opt. Soc. Am. B 31 2736-43

[44] Iaconis C and Walmsley I A 1999 Self-referencing spectral interferometry for measuring ultrashort optical pulses IEEE J. Quantum Electron. 35 501-9

[45] Miranda M, Fordell T, Arnold C, L'Huillier A and Crespo H 2012 Simultaneous compression and characterization of ultrashort laser pulses using chirped mirrors and glass wedges Opt. Express 20 688-97

[46] Xu B, Gunn J M, Cruz J M D, Lozovoy V V and Dantus M 2006 Quantitative investigation of the MIIPS method for phase measurement and compensation of femtosecond laser pulses J. Opt. Soc. Am. B 23 750-9 
[47] Steinmeyer G 2002 A review of ultrafast optics and optoelectronics J. Opt. A 5 R1-15

[48] Taft A et al 1996 Measurement of $10 \mathrm{fs}$ laser pulses IEEE J. Sel. Top. Quantum Electron. 2575

[49] Diels J-C, Fontaine J J, McMichael I C and Simoni F 1985 Control and measurement of ultrashort pulse shapes (in amplitude and phase) with femtosecond accuracy Appl. Opt. 24 1270-82

[50] Birkholz S, Steinmeyer G, Koke S, Gerth D, Bürger S and Hofmann B 2015 Phase retrieval via regularization in selfdiffraction-based spectral interferometry J. Opt. Soc. Am. B 32 983-92

[51] Balciunas $\mathrm{T}$ et al 2014 A strong-field driver in the singlecycle regime based on self-compression in a kagome fibre Nat. Commun. 66117

[52] Zewail A H 2000 Femtochemistry Angew. Chem., Int. Ed. Engl. 39 2586-631

[53] Suzuki T 2012 Time-resolved photoelectron spectroscopy of non-adiabatic electronic dynamics in gas and liquid phases Int. Rev. Phys. Chem. 31 265-318

[54] Mooney C R S, Horke D A, Chatterley A S, Simperler A, Fielding H H and Verlet J R R 2013 Taking the green fluorescence out of the protein: dynamics of the isolated GFP chromophore anion Chem. Sci. 4 921-7

[55] Gessner O et al 2006 Femtosecond multidimensional imaging of a molecular dissociation Science 311 219-22

[56] Conyard J, Addison K, Heisler I A, Cnossen A, Browne W R, Feringa B L and Meech S R 2012 Ultrafast dynamics in the power stroke of a molecular rotary motor Nat. Chem. 4 547-51

[57] Orr-Ewing A J 2014 Perspective: bimolecular chemical reaction dynamics in liquids J. Chem. Phys. 140090901

[58] Janssen M H M and Powis I 2014 Detecting chirality in molecules by imaging photoelectron circular dichroism Phys. Chem. Chem. Phys. 16 856-71

[59] Chergui M 2014 Introductory lecture: emerging photon technologies for chemical dynamics Faraday Discuss. 171 $11-40$

[60] Tenboer J et al 2014 Time-resolved serial crystallography captures high-resolution intermediates of photoactive yellow protein Science 346 1242-6

[61] Minitti M P et al 2015 Imaging molecular motion: femtosecond x-ray scattering of an electrocyclic chemical reaction Phys. Rev. Lett. 114255501

[62] Udem T, Reichert J, Holzwarth R and Hänsch T W 1999 Absolute optical frequency measurement of the cesium $D_{1}$ line with a mode-locked laser Phys. Rev. Lett. 823568

[63] Del'Haye P, Schliesser A, Arcizet O, Wilken T, Holzwarth R and Kippenberg T J 2007 Optical frequency comb generation from a monolithic microresonator Nature 450 1214-7

[64] Kourogi M, Widiyatomoko B, Takeuchi Y and Ohtsu M 1995 Limit of opticale to material dispersion IEEE J. Quantum Electron. 312120

[65] Ushijima I, Takamoto M, Das M, Ohkubo T and Katori T 2015 Cryogenic optical lattice clocks Nat. Photon 9185 Nicholson T L, Campbell S L, Hutson R B, Marti G E, Bloom B J, McNally R L, Zhang W, Barrett M D, Safronova M S, Strouse G F, Tew W L and Ye J 2015 Systematic evaluation of an atomic clock at $2 \times 10^{-18}$ total uncertainty Nat. Commun. 6 6896/1

[66] Wilken T, Lo Curto G, Probst R A, Steinmetz T, Manescau A, Pasquini L, Gonzalez Hernandez J I, Rebolo R, Hänsch T W, Udem T and Holzwarth R 2012 A spectrograph for exoplanet observations calibrated at the centimetre-per-second level Nature 485611

[67] Schliesser A, Picque N and Hänsch T W 2012 Mid-infrared frequency combs Nat. Photon. 6 440-9
[68] Gohle C, Udem T, Herrmann M, Rauschenberger J, Holzwarth R, Schuessler H A, Krausz F and Hansch T W 2005 A frequency comb in the extreme ultraviolet Nature 436234

Jones R J, Moll K D, Thorpe M J and Ye J 2005 Phasecoherent frequency combs in the vacuum ultraviolet via high-harmonic generation inside a femtosecond enhancement cavity Phys. Rev. Lett. 94193201

[69] Herr T, Brasch V, Jost J D, Wang C Y, Kondratiev N M, Gorodetsky M L and Kippenberg T J 2014 Temporal solitons in optical microresonators Nat. Photon. 8145

[70] Giunta M, Hänsel W, Beha K, Fischer M, Lezius M and Holzwarth R 2016 Ultra Low Noise Er:fiber Frequency Comb Comparison Conf. on Lasers and Electro-Optics OSA Technical Digest (Washington DC: Optical Society of America, 2016) paper STh4H.1

[71] Lezius M, Giunta M, Deutsch Ch, Wilken T, Hänsch T W, Kohfeldt A, Wicht A, Schkolnik V, Krutzik M, Duncker H, Hellmig O, Windpassinger P, Sengstock K, Peters A and Holzwarth R 2016 Frequency Combs for Space Applications Conf. on Lasers and Electro-Optics OSA Technical Digest (Washington, DC: Optical Society of America) paper STh4H.5

[72] Khurgin J B, Dikmelik Y, Hugi A and Faist J 2014 Coherent frequency combs produced by self frequency modulation in quantum cascade lasers Appl. Phys. Lett. 104081118 Wang C Y, Kuznetsova L, Gkortsas V M, Diehl L, Kärtner F X, Belkin M A, Belyanin A, Li X, Ham D, Schneider H, Grant P, Song C Y, Haffouz S, Wasilewski Z R, Liu H C and Capasso F 2009 Mode-locked pulses from mid-infrared Quantum Cascade Lasers Opt. Express 17 12929-43

[73] Kafka J D, Hall D W and Baer T 1989 Mode-locked erbiumdoped fiber laser with soliton pulse shaping Opt. Lett. 14 1269-71

[74] Duling I N 1991 All-fiber soliton laser mode locked with a nonlinear mirror Opt. Lett. 16 539-41

[75] Fu L, McKay H A and Dong L 2009 Extremely large mode area optical fibers formed by thermal stress Opt. Express 17 11782-93

[76] Stutzki F, Jansen F, Liem A, Jauregui C, Limpert J and Tünnermann A 201226 mJ, $130 \mathrm{~W}$ Q-switched fiber-laser system with near-diffraction-limited beam quality $O p t$. Lett. 37 1073-5

[77] Dong L 2008 Approximate treatment of the nonlinear waveguide equation in the regime of nonlinear self-focus $J$. Lightwave Technol. 26 3476-85

[78] Li C, Ma Y, Gao X, Niu F, Jiang T, Wang A and Zhang Z $20151 \mathrm{GHz}$ repetition rate femtosecond Yb:fiber laser for direct generation of carrier-envelope offset frequency Appl. Opt. 54 8350-3

[79] Chen H-W, Chang G, Xu S, Yang Z and Kärtner F X 2012 $3 \mathrm{GHz}$, fundamentally mode-locked, femtosecond $\mathrm{Yb}$-fiber laser Opt. Lett. 37 3522-4

[80] Kitamura R, Pilon L and Jonasz M 2007 Optical constants of silica glass from extreme ultraviolet to far infrared at near room temperature Appl. Opt. 46 8118-33

[81] Woodward R I, Howe R C T, Hu G, Torrisi F, Zhang M, Hasan T and Kelleher E J R 2015 Few-layer $\mathrm{MoS}_{2}$ saturable absorbers for short-pulse laser technology: current status and future perspectives Photon. Res. 3 A $30-41$

[82] Hanensel W, Holzwarth R, Doubek R, Mei M and Engelbrecht M 2014 Laser with nonlinear optical loop mirror US Patent Appl. No. $12 \uparrow / \uparrow 477,308$

[83] Lefrançois S, Wise F W, Sosnowski T S, Liu C-H and Galvanauskas A 2011 Scaling mode-locked fiber lasers to high energies using chirally-coupled core fiber CLEO Paper CMS7 
[84] Ding E, Lefrancois S, Kutz J N and Wise F W 2011 Scaling fiber lasers to large mode area: an investigation of passive mode-locking using a multi-mode fiber IEEE J. Quantum Electron. 47 597-606

[85] Mourou G A, Hulin D and Galvanauskas A 2006 The road to high peak power and high average power lasers: coherent-amplification network (CAN) AIP Conf. Proc. 827 152-63

[86] Mourou G, Brocklesby B, Tajima T and Limpert J 2013 The future is fibre accelerators Nat. Photon. 7 258-61

[87] Martin W S and Chernoch J P 1972 Multiple internal reflection face pumped laser US Patent 3,633,126

[88] Du K, Wu N, Xu J, Giesekus J, Loosen P and Poprawe R 1998 Partially end-pumped Nd:YAG slab laser with a hybrid resonator Opt. Lett. 23 370-2

[89] Wang Y Y, Peng X, Alharbi M, Dutin C F, Bradley T D, Gérôme F, Mielke M, Booth T and Benabid F 2012 Design and fabrication of hollow-core photonic crystal fibers for high-power ultrashort pulse transportation and pulse compression Opt. Lett. 37 3111-3

[90] Kippenberg T J, Holzwarth R and Diddams S A 2011 Microresonator-based optical frequency combs Science 332 $555-9$

[91] Arcizet O, Schliesser A, Del'Haye P, Holzwarth R and Kippenberg T J 2009 Optical frequency comb generation in monolithic microresonators Practical Applications of Microresonators (Boca Raton, FL: CRC press) ch 11, pp 483-506

[92] Del'Haye P, Coillet A, Loh W, Beha K, Papp S B and Diddams S A 2015 Phase steps and resonator detuning measurements in microresonator frequency combs Nat. Commun. 65668

[93] Brasch V, Geiselmann M, Herr T, Lihachev G, Pfeiffer M H P, Gorodetsky M L and Kippenberg T J 2016 Photonic chip-based optical frequency comb using soliton Cherenkov radiation Science 351 357-60

[94] Saha K, Okawachi Y, Shim B, Levy J S, Salem R, Johnson A R, Foster M A, Lamont M R E, Lipson M and Gaeta A L 2013 Modelocking and femtosecond pulse generation in chip-based frequency combs Opt. Express 21 1335-43

[95] Yi X, Yang Q F, Yang K Y, Suh M G and Vahala K 2015 Soliton frequency comb at microwave rates in a high-Q silica microresonator Optica 2 1078-85

[96] Jost J D, Herr T, Lecaplain C, Brasch V, Pfeiffer M H P and Kippenberg T J 2015 Counting the cycles of light using a self-referenced optical microresonator Optica 2 706-11

[97] Del'Haye P, Coillet A, Fortier T, Beha K, Cole D C, Yang K Y, Lee H, Vahala K J, Papp S B and Diddams S A 2016 Phase-coherent microwave-to-optical link with a selfreferenced microcomb Nat. Photon 10 516-20

[98] Brasch V, Lucas E, Jost J D, Geiselmann M and Kippenberg T J 2016 Self-referencing of an on-chip soliton Kerr frequency comb without external broadening (arXiv:1605.02801)

[99] Herr T, Brasch V, Jost J D, Mirgorodskiy I, Lihachev G, Gorodetsky M L and Kippenberg T J 2014 Mode spectrum and temporal soliton formation in optical microresonators Phys. Rev. Lett. 113123901

[100] Del'Haye P, Beha K, Papp S B and Diddams S A 2014 Selfinjection locking and phase-locked states in microresonatorbased optical frequency combs Phys. Rev. Lett. 112043905

[101] Wu R, Torres-Company V, Leaird D E and Weiner A M 2013 Supercontinuum-based 10-GHz flat-topped optical frequency comb generation Opt. Express 21 6045-52

[102] Del'Haye P 2011 Optical frequency comb generation in monolithic microresonators PhD Thesis LMU Munich

[103] Riemensberger J, Hartinger K, Herr T, Brasch V, Holzwarth R and Kippenberg T J 2012 Dispersion engineering of thick high-Q silicon nitride ring-resonators via atomic layer deposition Opt. Express 20 27661-9

[104] Grudinin I S and Yu N 2015 Dispersion engineering of crystalline resonators via microstructuring Optica 2 221-4

[105] Yang K, Beha K, Cole D, Yi X, Del'Haye P, Lee H, Li J, Oh D, Diddams S, Papp S and Vahala K 2015 Broadband dispersion engineered microresonator on-a-chip Nat. Photon. 10 316-20

[106] Druon F, Balembois F and Georges P 2007 New laser crystals for the generation of ultrashort pulses $C$. ${ } R$. Phys. 8 153-64

[107] Keller U, Weingarten K J, Kärtner F X, Kopf D, Braun B, Jung I D, Fluck R, Hönninger C, Matuschek N and Aus der Au J 1996 Semiconductor saturable absorber mirrors (SESAMs) for femtosecond to nanosecond pulse generation in solid-state lasers IEEE J. Sel. Top. Quantum Electron. 2 435-53

[108] Südmeyer T, Marchese S V, Hashimoto S, Baer C R E, Gingras G, Witzel B and Keller U 2008 Femtosecond laser oscillators for high-field science Nat. Photon. 2 599-604

[109] Giesen H, Hügel A, Voss K, Wittig U, Brauch and Opower H 1994 Scalable concept for diode-pumped high-power solidstate lasers Appl. Phys. B 58 365-72

[110] Saraceno J, Emaury F, Heckl O H, Baer C R E, Hoffmann M, Schriber C, Golling M, Südmeyer T and Keller U 2012 $275 \mathrm{~W}$ average output power from a femtosecond thin disk oscillator operated in a vacuum environment Opt. Express 2023535

[111] Russbueldt P, Mans T, Weitenberg J, Hoffmann H D and Poprawe R 2010 Compact diode-pumped $1.1 \mathrm{~kW}$ Yb:YAG Innoslab femtosecond amplifier Opt. Lett. 34 4169-71

[112] Negel J P, Voss A, Ahmed M A, Bauer D, Sutter D, Killi A and Graf T $20131.1 \mathrm{~kW}$ average output power from a thin-disk multipass amplifier for ultrashort laser pulses Opt. Lett. 38 5442-5

[113] Keller U and Tropper A C 2006 Passively modelocked surface-emitting semiconductor lasers Phys. Rep. 429 $67-120$

[114] Maas J H C, Bellancourt A-R, Rudin B, Golling M, Unold H J, Südmeyer T and Keller U 2007 Vertical integration of ultrafast semiconductor lasers Appl. Phys. B 88 493-7

[115] Mourou G, Mironov S, Khazanov E and Sergeev A 2014 Single cycle thin film compressor opening the door to zeptosecond-exawatt physics Eur. Phys. J. Spec. Top. 223 $1181-8$

[116] Naumova N M, Nees J A, Sokolov I V, Hou B and Mourou G A 2004 Relativistic generation of isolated attosecond pulses in a $\lambda^{3}$ focal volume Phys. Rev. Lett. 92 3-6

[117] Tajima T 2014 Laser acceleration in novel media Eur. Phys. J. Spec. Top. 223 1037-44

[118] Mourou G A, Barry C P J and Perry M D 1998 Ultrahighintensity lasers: physics of the extreme on a tabletop Phys. Today $\mathbf{5 1} 22$

[119] Braun A, Korn G, Liu X, Du D, Squier J and Mourou G 1995 Self-channeling of high-peak-power femtosecond laser pulses in air Opt. Lett. 2073

[120] Popmintchev T et al 2012 Bright coherent ultrahigh harmonics in the $\mathrm{keV} \mathrm{X}$-ray regime from mid-infrared femtosecond lasers Science 3361287

[121] Clerici M et al 2013 Wavelength scaling of terahertz generation by gas ionization Phys. Rev. Lett. 110253901

[122] Rohwetter P et al 2010 Laser-induced water condensation in air Nat. Photon. 4451

[123] Wright L G, Christodoulides D N and Wise F W 2015 Controllable spatiotemporal nonlinear effects in multimode fibres Nat. Photon. 9306 
[124] Jang J K, Erkintalo M, Murdoch s G and Coen S 2013 Ultraweak long-range interactions of solitons observed over astronomical distances Nat. Photon. 7657

[125] Gea-Banacloche J 1989 Two-photon absorption of nonclassical light Phys. Rev. Lett. 621603

[126] Solli D R, Ropers C, Koonath P and Jalali B 2007 Optical rogue waves Nature $\mathbf{4 5 0} 1054$

[127] Carusotto I and Ciuti C 2013 Quantum fluids of light Rev. Mod. Phys. 85299

[128] Philbin T G, Kuklewicz C, Robertson S, Hill S, König F and Leonhardt U 2008 Fiber-optical analog of the event horizon Science 3191367

[129] Kreuzer L B and Patel C K N 1971 Nitric oxide air pollution-detection by optoacoustic spectroscopy Science 17345

[130] Yao Y, Hoffman A J and Gmachl C F 2012 Mid-infrared quantum cascade lasers Nat. Photon. 6 432-9

[131] Tittel F K, Richter D and Fried A 2003 Mid-infrared laser applications in spectroscopy Solid-State Mid-Infrared Laser Sources 89 445-510

[132] Udem T, Holzwarth R and Hansch T W 2002 Optical frequency metrology Nature 416 233-7

[133] Hugi A et al 2012 Mid-infrared frequency comb based on a quantum cascade laser Nature 492 229-33

[134] Mandon J, Guelachvili G and Picque N 2009 Fourier transform spectroscopy with a laser frequency comb Nat. Photon. 3 99-102

[135] Schiller S 2002 Spectrometry with frequency combs Opt. Lett. 27 766-8

[136] Jin Y, Cristescu S M, Harren F J M and Mandon J 2015 Femtosecond optical parametric oscillators toward real-time dual-comb spectroscopy Appl. Phys. B 119 65-74
[137] Giordamaine J A and Miller R C 1965 Tunable coherent parametric oscillation in $\mathrm{LiNbO}_{3}$ at optical frequencies Phys. Rev. Lett. 14 973-6

[138] Cerullo G and De Silvestri S 2003 Ultrafast optical parametric amplifiers Rev. Sci. Instrum. 74 1-18

[139] Brida D, Manzoni C, Cirmi G, Marangoni M, Bonora S, Villoresi P, De Silvestri S and Cerullo G 2010 Few-opticalcycle pulses tunable from the visible to the mid-infrared by optical parametric amplifiers J. Opt. 12013001

[140] Krebs M, Hädrich S, Demmler S, Rothhardt J, Zaïr A, Chipperfield L, Limpert J and Tünnermann A 2013 Towards isolated attosecond pulses at megahertz repetition rates Nat. Photon. 7 555-9

[141] Dubietis A, Jonusauskas G and Piskarskas A 1992 Powerful femtosecond pulse generation by chirped and stretched pulse parametric amplification in BBO crystal Opt. Commun. 88 437-40

[142] Herrmann D, Veisz L, Tautz R, Tavella F, Schmid K, Pervak V and Krausz F 2009 Generation of sub-three-cycle, 16 TW light pulses by using noncollinear optical parametric chirped-pulse amplification Opt. Lett. 34 2459-61

[143] Baltŭska A, Fuji T and Kobayashi T 2002 Controlling the carrier-envelope phase of ultrashort light pulses with optical parametric amplifiers Phys. Rev. Lett. 88133901

[144] Manzoni C, Mücke O D, Cirmi G, Fang S, Moses J, Huang S-W, Hong K-H, Cerullo G and Kärtner F X 2015 Coherent pulse synthesis: towards sub-cycle optical waveforms Laser Photon. Rev. 9 129-71

[145] Hemmer M, Sánchez D, Jelínek M, Smirnov V, Jelinkova H, Kubeček V and Biegert J 2015 2- $\mu \mathrm{m}$ wavelength, highenergy Ho:YLF chirped-pulse amplifier for mid-infrared OPCPA Opt. Lett. 40 451-4 